This version was created by the author in August, 2013.

The final publication is available at https://link.springer.com/; namely, at https://link.springer.com/content/pdf/10.1007\%2Fs00012-014-0294-z.pdf

Algebra Universalis 72 (2014) 125-154; $\quad$ DOI 10.1007/s00012-014-0294-Z

\title{
Patch extensions and trajectory colorings of slim rectangular lattices
}

\section{GÁBor CzÉDli}

ABSTRACT. With the help of our new tools in the title, we give an efficient representation of the congruence lattice of a slim rectangular lattice by an easy-to-visualize quasiordering on the set of its meet-irreducible elements or, equivalently, on the set of its trajectories.

\section{Introduction}

For the key definitions, see Section 2. Unless otherwise stated, all lattices in this paper are finite.

In the paper, our first goal is to generalize the fork extensions of slim semimodular lattices, introduced by G. Czédli and E. T. Schmidt [12], to patch extensions and in particular, multi-fork extensions. Multi-fork extensions lead to a new structural description of slim rectangular lattices, see Theorem 3.7. Based on multi-fork extensions, our the second goal is to associate an easyto-visualize quasi-coloring with a slim rectangular lattice $L$, which we call the trajectory quasi-coloring of $L$. The trajectory quasi-coloring induces a coloring, called the trajectory coloring of $L$. This coloring gives the ordered set of join-irreducible congruences of $L$ and, therefore, determines the congruence lattice of $L$. The main result, Theorem 7.3, describes the trajectory coloring of $L$ explicitely. This theorem will probably be useful in characterizing the class of congruence lattices of slim semimodular (or slim patch) lattices; this problem was raised in G. Grätzer [20].

1.1. Outline. Section 2 gives an overview of slim and rectangular semimodular lattices, their trajectories, and their congruences. Section 3 defines patch and multi-fork extensions, and points out in Theorem 3.7 that each rectangular

2010 Mathematics Subject Classification: 06C10.

Key words and phrases: Rectangular lattice, patch lattice, slim semimodular lattice, congruence lattice, lattice coloring, quasi-coloring, quasiordering, fork extension, multi-fork extension, patch extension.

This research was supported by the European Union and co-funded by the European Social Fund under the project "Telemedicine-focused research activities on the field of Mathematics, Informatics and Medical sciences" of project number "TÁMOP-4.2.2.A11/1/KONV-2012-0073", and by NFSR of Hungary (OTKA), grant number K83219. 
lattice can be obtained from the direct product of two chains by multi-fork extensions at distributive 4-cells. Section 4 introduces trajectory quasi-colorings. By Theorem 4.4 of this section, trajectory quasi-colorings of slim rectangular lattices are quasi-colorings, that is, appropriate tools to describe the congruence lattices of these lattices. Section 5 proves Theorem 4.4. Theorem 5.5, also called the multi-fork theorem, is of separate interest. Section 6 generalizes the multi-fork theorem and its auxiliary "retraction lemma" (Lemma 5.3) from multi-fork extensions to patch extensions. The rest of the paper does not rely on this section. In Section 7, we turn Theorem 4.4 into our main result, Theorem 7.3, which describes a real coloring, the trajectory coloring (not just a quasi-coloring) of a slim rectangular lattice. Finally, Section 8 contains some comments on possible generalizations.

1.2. Historical background. A finite lattice $L$ is slim, if $\mathrm{Ji} L$, the set of nonzero join-irreducible elements of $L$, is included in the union of two chains of $L$; see G. Czédli and E. T. Schmidt [11]. In the semimodular case, this concept was first introduced by G. Grätzer and E. Knapp [21] in a different way. The theory of slim semimodular lattices has developed a lot recently, as witnessed by G. Czédli [1], [3], [4], [5], and [6], G. Czédli, T. Dékány, L. Ozsvárt, N. Szakács, and B. Udvari [7], G. Czédli and G. Grätzer [8] and [9], G. Czédli, L. Ozsvárt, and B. Udvari [10], G. Czédli and E. T. Schmidt [11], [12], [13], and [14], G. Grätzer [18], [20], G. Grätzer and E. Knapp [21], [22], [23], and [24], G. Grätzer and E. T. Schmidt [26], and E. T. Schmidt [29]. Note that [11] gives an application of these lattices outside lattice theory. [1], [5], $[8],[12],[13],[14],[18]$, and [21], partly of fully, are devoted to their structural descriptions. While [12] describes these lattices with fork extensions, [18] does the same with patch lattices.

The present paper combines fork extensions and patch lattices to define patch extensions and, in particular, multi-fork extensions.

Influenced by G. Grätzer [16] and E. T. Schmidt [29], quasi-coloring was introduced in G. Czédli [3]. This is an efficient tool to describe the congruence lattice of a finite lattice. Its advantage is explained in Subsection 4.1 here and in the subsection "Method" of [3]. Here, we introduce a quasi-coloring, called trajectory quasi-coloring, of a slim rectangular lattice. We use multi-fork extensions to prove that it is a quasi-coloring.

1.3. Terminology. Unless otherwise stated, we follow the standard terminology and notation of lattice theory; see, for example, G. Grätzer [17]. Ordered sets are nonempty sets equipped with orderings, that is, with reflexive, transitive, antisymmetric relations. Note that an ordered set is often called a partially ordered set, poset, or an order. 


\section{Some basic concepts from lattice theory}

For an overview of these concepts, see also G. Czédli and G. Grätzer [9].

2.1. Planar semimodular lattices. It is proved in G. Czédli and E. T. Schmidt [11, Lemmas 5 and 6], or in G. Czédli and E. T. Schmidt [12, Proposition 5], that slim lattices are planar; for slim semimodular lattices this was proved earlier in G. Grätzer and E. Knapp [21]. In this paper, a lattice diagram is a planar Hasse diagram of a finite lattice. Assume that $D_{1}$ and $D_{2}$ are lattice diagrams. A bijection $\varphi: D_{1} \rightarrow D_{2}$ is a similarity map if it is a lattice isomorphism preserving the left-right order of (upper) covers and lower covers of an element of $D_{1}$. If there is a similarity map $D_{1} \rightarrow D_{2}$, then these two lattice diagrams are similar, and we will treat them as equal. Hence, a finite planar lattice has only finitely many diagrams. If $D$ is a lattice diagram of a planar lattice $L$, then lattice theoretical concepts also apply to $D$. If a lattice property is used for a lattice diagram, then we often say "diagram" instead of "lattice diagram".

The edges of a planar lattice diagram $D$ divide the plane into regions. A minimal (necessarily non-empty) region is called a cell, a four-element cell is a 4-cell; it is also a covering square, that is, cover-preserving four-element Boolean sublattice of $D$. For example, the usual diagram of $M_{3}$ has exactly two 4-cells and three covering squares. A 4-cell $H$ of $D$ consists of its bottom, $0_{H}$, top, $1_{H}$, left corner, $\operatorname{lc}(H)$, and right corner, $\operatorname{rc}(H)$. If $\downarrow 1_{H}=\{x \in D$ : $\left.x \leq 1_{H}\right\}$ is slim or distributive, then $H$ is a slim 4-cell or a distributive 4cell, respectively. The left boundary chain and the right boundary chain of $L$ are denoted by $\mathrm{C}_{\ell}(D)$ and $\mathrm{C}_{\mathrm{r}}(D)$, respectively, while their union, $\operatorname{Bnd}(D)$, is the boundary of $D$. (Upper case acronyms define sets, lower case acronyms, elements.) The set $D \backslash \operatorname{Bnd}(D)$ is the interior of $D$, and its members are the interior elements.

For the sake of mathematical rigor, note that many visual concepts, such as an element is on the left of another element, are exactly defined in D. Kelly and I. Rival [28]; see also G. Czédli and G. Grätzer [9]. Also, we shall distinguish lattice properties and concepts, which do not depend on the planar diagram chosen, from diagram properties and concepts, which are diagram dependent. For example, $\operatorname{lc}(H)$ is a diagram concept, a covering square is a lattice concept, and a 4-cell is a diagram concept for $\mathrm{M}_{3}$ but it a lattice concept for every slim semimodular lattice by G. Czédli and E. T. Schmidt [11, Lemma 2.3].

2.2. Trajectories. For a slim semimodular lattice $L$, let $\operatorname{PrInt}(L)$ denote the set of edges, that is prime intervals, of $L$. Similarly, $\operatorname{Int}(L)$ denotes the set of intervals of $D$. For $\mathfrak{p}$ and $\mathfrak{q} \in \operatorname{PrInt}(L), \mathfrak{p}$ and $\mathfrak{q}$ are consecutive if they are opposite sides of a 4-cell. Following G. Czédli and E. T. Schmidt [11, Lemma 2.3], maximal sequences of consecutive prime intervals form a trajectory. In other words, if $\sim^{\text {traj }}$ denotes the transitive reflexive closure of the relation of 


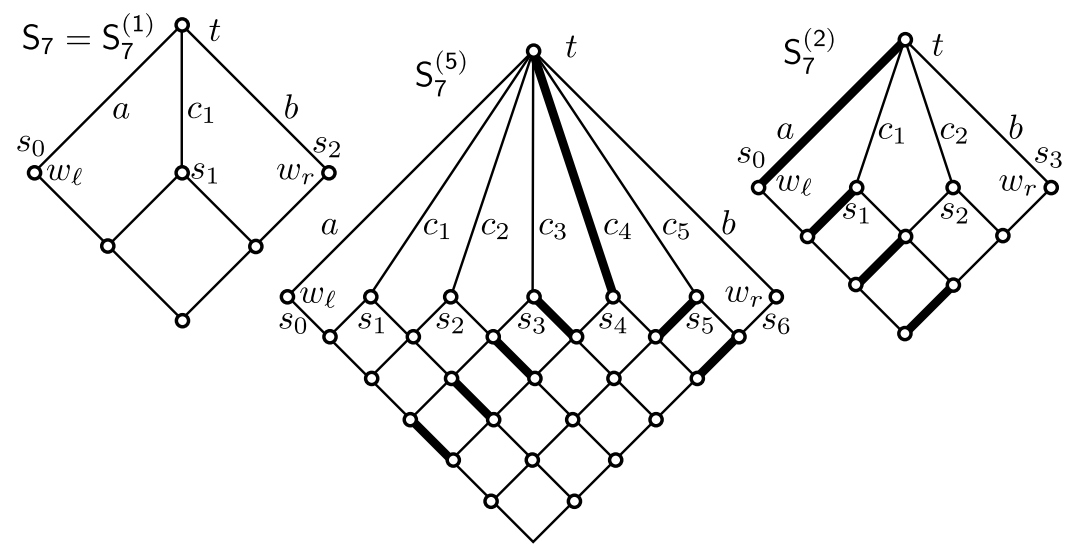

FIGURE 1. Some of the $\mathrm{S}_{7}^{(n)}$; note that $w_{\ell}=s_{0}$ and $w_{r}=s_{n+1}$

being consecutive on $\operatorname{PrInt}(L)$, then a trajectory is a block of the equivalence relation $\sim^{\text {traj }}$. For example, a trajectory of $S_{7}^{(2)}$ and that of $S_{7}^{(5)}$ are indicated in Figure 1 by thick edges.

Next, we fix a diagram $D \in \operatorname{PrInt}(L)$, and recall the basic properties of trajectories from G. Czédli and E.T. Schmidt [11] with some new features. Unless otherwise stated, a trajectory starts with an edge in the left boundary chain $\mathrm{C}_{\ell}(D)$, goes from left to right, and ends in $\mathrm{C}_{\mathrm{r}}(D)$. Trajectories do not branch out. Consecutive edges of a trajectory form 4-cells; these 4-cells are the 4-cells of the trajectory. An up-trajectory goes up while a down-trajectory goes down, making no turn. These two types of trajectories are called straight trajectories. For example, the trajectory of $\mathrm{S}_{7}^{(2)}$ in Figure 1 is a (straight) down-trajectory. A hat-trajectory is a non-straight trajectory that goes up first, at least one step, then turns to the lower right, and finally it goes down, at least one step. For example, a hat trajectory of $S_{7}^{(5)}$ is depicted in Figure 1. We know from [11] that there are no more types of trajectories; in particular,

a trajectory can make only one turn, a down turn.

2.3. Rectangular lattices. The elements of $\operatorname{Bnd}(D) \cap \mathrm{Ji} D \cap \mathrm{Mi} D$ are called the weak corners of $D$. For $a \in \mathrm{Ji} L$ and $b \in \mathrm{Mi} L$, the unique upper cover of $a$ and the unique lower cover of $b$ are denoted by $a^{*}$ and $b_{*}$, respectively. A corner is defined as a weak corner $d$ such that $d^{*}$ has exactly two lower covers and $d_{*}$ has exactly two covers. Corners and weak corners of $D$ are left or right. Following G. Grätzer and E. Knapp [21], a planar lattice diagram $D$ is rectangular if it is semimodular, $\mathrm{C}_{\ell}(D)$ has exactly one weak corner, $\operatorname{lc}(D), \mathrm{C}_{\mathrm{r}}(D)$ has exactly one weak corner, $\operatorname{rc}(D)$, and these two elements are complementary, that is, $\operatorname{lc}(D) \wedge \operatorname{rc}(D)=0$ and $\operatorname{lc}(D) \vee \operatorname{rc}(D)=1$. If, in addition, $\operatorname{lc}(D)$ and $\operatorname{rc}(D)$ are coatoms, then $D$ is a patch diagram, see G. Czédli and E. T. Schmidt [14]. If a lattice $L$ has a rectangular diagram or a patch 
diagram, then $L$ is a rectangular lattice or a patch lattice, respectively. We know from G. Czédli and E. T. Schmidt [14, Lemma 4.9] that if one diagram of a planar semimodular lattice is rectangular or patch, then so are all of its diagrams. For example, $\mathrm{S}_{7}=\mathrm{S}_{7}^{(1)}, \mathrm{S}_{7}^{(2)}$, and $\mathrm{S}_{7}^{(5)}$ in Figure 1 are slim patch diagrams, and so are the $S_{7}^{(n)}$ for all $n \in \mathbb{N}=\{1,2,3, \ldots\}$. The definition of $\mathrm{S}_{7}^{(n)}$ should be clear from the examples: take the usual diagram of $C_{n+2} \times C_{n+2}$ where $C_{n+2}$ denotes the $(n+2)$-element chain, and, with the exception of 1 , delete all elements with height greater than $n+1$.

For a rectangular lattice diagram $D$, the intervals $\mathrm{C}_{\ell \ell}(D)=[0, \operatorname{lc}(D)]$, $\mathrm{C}_{\ell \mathrm{r}}(D)=[0, \operatorname{rc}(D)], \mathrm{C}_{\mathrm{u} \ell}(D)=[\operatorname{lc}(D), 1]$, and $\mathrm{C}_{\mathrm{ur}}(D)=[\operatorname{rc}(D), 1]$ are chains and subsets of the boundary by G. Grätzer and E. Knapp [23]. These chains are called the lower left boundary (chain), the lower right boundary, the upper left boundary, and the upper right boundary of $D$, respectively.

2.4. Congruence spreading. By folklore, see G. Grätzer [16, Sect. I.3.2]

$$
\operatorname{Ji}(\operatorname{Con} M)=\{\operatorname{con}(\mathfrak{p}): \mathfrak{p} \in \operatorname{PrInt}(M)\}
$$

holds for every finite lattice $M$. Let $\mathfrak{p}_{1}=\left[x_{1}, y_{1}\right]$ and $\mathfrak{p}_{2}=\left[x_{2}, y_{2}\right]$ be intervals of $M$. Following the terminology and notation of G. Grätzer [17], if $y_{1} \vee x_{2}=y_{2}$ and $x_{1} \leq x_{2}$, then we say that $\mathfrak{p}_{1}$ is up congruence-perspective to $\mathfrak{p}_{2}$, in notation $\mathfrak{p}_{1} \stackrel{\text { up }}{\rightarrow} \mathfrak{p}_{2}$. Similarly, if $x_{1} \wedge y_{2}=x_{2}$ and $y_{1} \geq y_{2}$, then $\mathfrak{p}_{1}$ is down congruence perspective to $\mathfrak{p}_{2}$, in notation $\mathfrak{p}_{1} \stackrel{\text { dn }}{\rightarrow} \mathfrak{p}_{2}$. If $\mathfrak{p}_{1} \stackrel{\text { up }}{\rightarrow} \mathfrak{p}_{2}$ or $\mathfrak{p}_{1} \stackrel{\text { dn }}{\rightarrow} \mathfrak{p}_{2}$, then the interval $\mathfrak{p}_{1}$ is congruence-perspective to the interval $\mathfrak{p}_{2}$; in formula, $\mathfrak{p}_{1} \rightarrow \mathfrak{p}_{2}$. The transitive closure of congruence-perspectivity is called congruence-projectivity. In this paper, it will be denoted by $\mathfrak{p} \Rightarrow \mathfrak{q}$. Sometimes we will use subscripts such as $\mathfrak{p}_{1} \rightarrow_{M} \mathfrak{p}_{2}$ and $\mathfrak{p} \Rightarrow M \mathfrak{q}$ to avoid ambiguity. We will often rely, usually implicitly, on the fact that

$$
\text { for } \mathfrak{p}, \mathfrak{q} \in \operatorname{Int}(M), \mathfrak{p} \Rightarrow \mathfrak{q} \text { iff } \operatorname{con}(\mathfrak{p}) \supseteq \operatorname{con}(\mathfrak{q}),
$$

see, e.g. G. Grätzer [16, Lemma I.3.6] or [17, Thm. 230], or see also G. Grätzer [15, Sect. III.1]. In particular, we say that $\mathfrak{p}$ and $\mathfrak{q}$ are congruence-equivalent if $\mathfrak{p} \Rightarrow \mathfrak{q}$ and $\mathfrak{q} \Rightarrow \mathfrak{p}$. Note that (2.3) holds even if $\mathfrak{p}$ or $\mathfrak{q}$ is a singleton interval. For $\mathfrak{p}, \mathfrak{q} \in \operatorname{PrInt}(L)$, we say that $\mathfrak{p}$ transposes up to $\mathfrak{q}$, or that $\mathfrak{q}$ transposes down to $\mathfrak{p}$, if $1_{\mathfrak{q}}=1_{\mathfrak{p}} \vee 0_{\mathfrak{q}}$ and $0_{\mathfrak{p}}=1_{\mathfrak{p}} \wedge 0_{\mathfrak{q}}$. In this case, $\mathfrak{p}$ and $\mathfrak{q}$ are transposed intervals. Obviously, transposed intervals are congruence-equivalent. Since consecutive prime intervals are transposed, all prime intervals of a trajectory in a slim semimodular lattice are congruence equivalent. This observation and (2.2) lead to the following principle.

Remark 2.1. To understand the congruence lattices of a slim semimodular lattice, it suffices to focus on its trajectories.

Since we often have to verify that an equivalence is actually a lattice congruence, the following lemma of G. Grätzer [19] will be quite useful. It would be hard to over-emphasize its importance. Since its proof is not difficult, it is surprising that the lemma has not been discovered earlier. 
Lemma 2.2 (G. Grätzer [19]). Assume that $\Theta$ is an equivalence on a lattice $L$ of finite length with intervals as equivalence blocks. Then $\Theta$ is a congruence iff the following condition and its dual hold: for any $x, y, z \in L$, if $\langle x, y\rangle \in \Theta$, $y \neq z, x \prec y$, and $x \prec z$, then $\langle z, y \vee z\rangle \in \Theta$.

\section{Patch extensions}

Definition 3.1. Let $P$ be a slim patch diagram, and assume that $H$ is a slim 4-cell of a slim semimodular lattice diagram $D$. We define a new lattice diagram $D[P \leadsto H]$, the patch extension of $D$ at the 4 -cell $H$ with the patch diagram $P$ as follows; see Figure 2 . Let $k=$ length $P$, and observe that $\mathrm{C}_{\ell \ell}(P) \cong \mathrm{C}_{\ell \mathrm{r}}(P) \cong \mathrm{C}_{\mathrm{k}}$. Let $A$ and $B$ be the trajectories containing the edge $\left\langle 0_{H}, \mathrm{C}_{\ell}(H)\right\rangle$ and the edge $\left\langle 0_{H}, \mathrm{C}_{\mathrm{r}}(H)\right\rangle$, respectively. Let the set of 4-cells of $A$ on the left of $\left\langle 0_{H}, \mathrm{C}_{\ell}(H)\right\rangle$ be denoted by ${ }^{\diamond} A$. Similarly, let $B^{\diamond}$ stand for the set of 4-cells of $B$ on the right of $\left\langle 0_{H}, \mathrm{C}_{\mathrm{r}}(H)\right\rangle$. First, we replace $H$ by $P$. Next, we replace each edge of $A$ on the left of $\left\langle 0_{H}, \mathrm{C}_{\ell}(H)\right\rangle$ by $\mathrm{C}_{\mathrm{k}}$ such that each 4-cell in ${ }^{\ominus} A$ is replaced by $C_{2} \times C_{k}$. Similarly, we replace each edge of $B$ on the right of $\left\langle 0_{H}, \mathrm{C}_{\mathrm{r}}(H)\right\rangle$ by $\mathrm{C}_{\mathrm{k}}$ such that each 4-cell in $B^{\diamond}$ is replaced by $\mathrm{C}_{2} \times \mathrm{C}_{\mathrm{k}}$. The diagram we have just obtained is $D[P \leadsto H]$. In Figure 2, the new elements, that is, the elements of $D[P \sim H] \backslash P$, are black-filled.

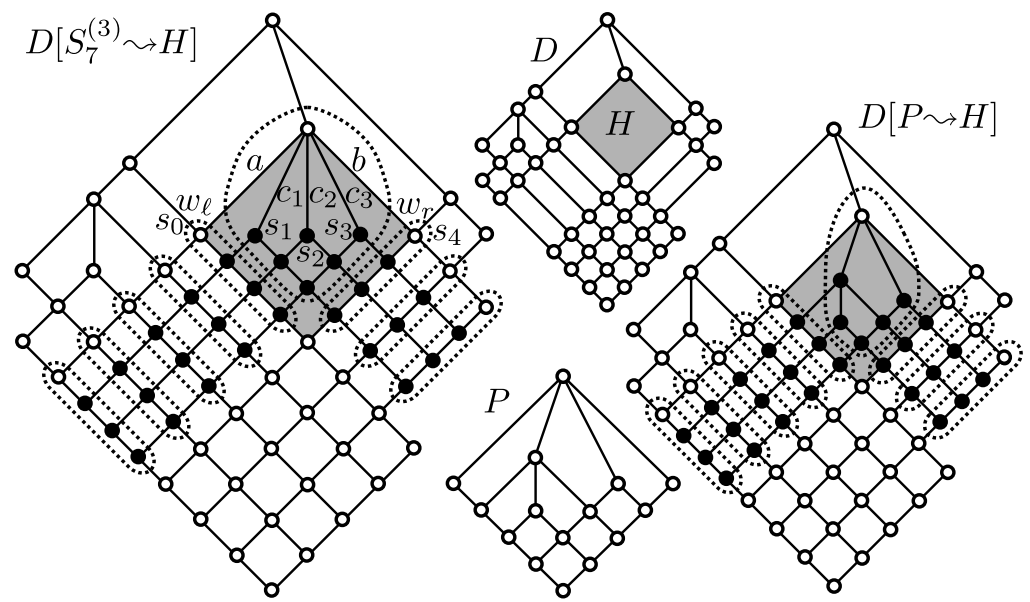

Figure 2. A multi-fork extension, $D\left[\mathrm{~S}_{7}^{(3)} \leadsto H\right]$, and a patch extension, $D[P \leadsto H]$

For $P=\mathrm{S}_{7}, D\left[\mathrm{~S}_{7} \leadsto H\right]$ is the (single) fork extension introduced in G. Czédli and E. T. Schmidt [12]. For $P=\mathrm{S}_{7}^{(n)}$, we call $D\left[\mathrm{~S}_{7}^{(n)} \leadsto H\right]$ the $n$-fold fork extension of $D$ at the 4-cell $H$; we speak of multi-fork extensions if $n$ is not specified. Fork extensions are the same as 1-fold fork extensions.

Remark 3.2. Since trajectories and 4-cells are lattice concepts for slim semimodular lattices, so is the multi-fork extension. However, the patch extension 
is not a lattice concept, because if we flip $P$ with respect to a vertical axis and keep $D$ unchanged, then usually we obtain a different lattice.

Proposition 3.3. Patch extensions and, in particular, multi-fork extensions of slim semimodular lattice diagrams are also slim semimodular lattice diagrams.

Proof. The particular case of (single) fork extensions is proved in G. Czédli and E. T. Schmidt [12, Theorem 11]. We know from G. Czédli and E. T. Schmidt [14, Theorem 3.4] that each slim patch diagram $P$ is obtained from a single 4-cell by a sequence of fork extensions. Hence, $D[P \sim H]$ can be obtained from $D$ by a sequence of (single) fork extensions. Thus our statement follows from this particular case.

Since multi-fork extensions are lattice concepts, diagrams could be replaced by lattices in Lemmas 3.4 and 3.6, Remark 3.5, and Theorem 3.7 below. In the rest of the paper, we are mostly interested in multi-fork and patch extensions at distributive 4-cells.

Lemma 3.4 (Commutativity of multi-fork extensions). Let $D$ be a slim semimodular lattice diagram with distributive 4-cells $H_{1}$ and $H_{2}$ such that their tops, $t_{1}=1_{H_{1}}$ and $t_{2}=1_{H_{2}}$, are incomparable.

(i) For $i \in\{1,2\}$, if we perform a multi-fork extension at $H_{i}$, then $H_{3-i}$ remains a distributive 4-cell.

(ii) Let $n_{1}, n_{2} \in \mathbb{N}$, and let $i \in\{1,2\}$. Then the $n_{3-i}$-fold fork extension at $H_{3-i}$ of the $n_{i}$-fold fork extension of $D$ at $H_{i}$ does not depend on the choice of $i \in\{1,2\}$.

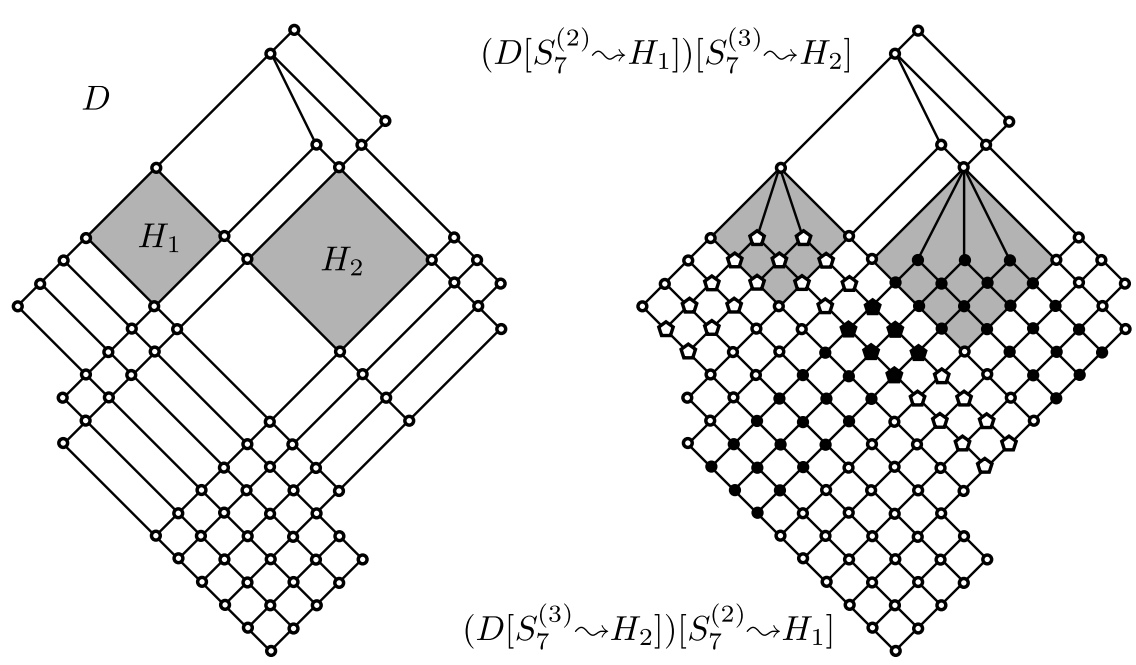

FigurE 3. $\left(D\left[\mathrm{~S}_{7}^{(2)} \leadsto H_{1}\right]\right)\left[\mathrm{S}_{7}^{(3)} \leadsto H_{2}\right]=\left(D\left[\mathrm{~S}_{7}^{(3)} \leadsto H_{2}\right]\right)\left[\mathrm{S}_{7}^{(2)} \leadsto H_{1}\right]$ 
Proof. The situation is illustrated in Figure 3, where $\left.D\left[\mathrm{~S}_{7}^{(2)}\right) \sim H_{1}\right]$ consists of the small empty circles, which are the elements of $D$, and of the somewhat bigger empty pentagons, while $\left.D\left[\mathrm{~S}_{7}^{(3)}\right) \leadsto H_{2}\right]$ consists of the little empty and the little black circles. We know from G. Czédli and E. T. Schmidt [12, Lemma 15] that a slim semimodular diagram is distributive if and only if it contains no cover-preserving $S_{7}$ sublattice. This proves the first part. Hence, Figure 3 is sufficiently general to imply the rest of Lemma 3.4.

Remark 3.5. It is straightforward to see that Part (ii) of Lemma 3.4 also holds if $t_{1}$ and $t_{2}$ are comparable, but we will not use this fact.

The following lemma is evident. Note that $H$ need not be distributive.

Lemma 3.6 (Transitivity of multi-fork extensions). Let $D$ be a slim semimodular diagram with a 4-cell $H$ with top $t=1_{H}$, and let $m, n \in \mathbb{N}$. If $D^{\prime}=D\left[\mathrm{~S}_{7}^{(m)} \leadsto H\right]$ and $H^{\prime}$ is a 4 -cell of $D^{\prime}$ whose top is $t$, then the equality $D^{\prime}\left[\mathbf{S}_{7}^{(n)} \leadsto H^{\prime}\right]=D\left[\mathbf{S}_{7}^{(m+n)} \leadsto H\right]$ holds.

The following statement does not have a "single fork" counterpart. Grids are the usual planar diagrams of $C_{m} \times C_{n}$ for $m, n \in\{2,3, \ldots\}$.

Theorem 3.7. Each slim rectangular lattice diagram is obtained from a grid by a sequence of multi-fork extensions at distributive 4-cells, and every diagram obtained this way is a slim rectangular diagram.

Proof. By Lemma 3.6, a multi-fork extension can be replaced by a sequence of single fork extensions. Hence, the second part of the statement follows from G. Czédli and E. T. Schmidt [14, Proposition 2.3].

For the sake of contradiction, suppose that the (first part of the) statement fails, and that $D$, a slim rectangular diagram, is a counterexample of minimum size. By G. Czédli and E. T. Schmidt [14, Proposition 2.4(i)], D, like every rectangular diagram, is obtained from a grid by a sequence of (single) fork extensions. There is at least one single fork extension since $D$ is a counterexample. Hence, having an $S_{7}$ sublattice, $D$ is not distributive. Therefore, we can choose an element $t \in D$ such that $\downarrow t$ is not distributive but $\downarrow t^{\prime}$ is distributive for all $t^{\prime}<t$. The combination of [12, Lemma 15] and [12, Proof of Lemma 22] contains the statement that $t$ is the top of a a cover-preserving $\mathrm{S}_{7}$ sublattice and also the top of a strong fork; this concept is defined in [12] but we do not need it. In our terminology, this statement says that there is a rectangular diagram $D^{\prime}$ containing $t$ and a 4-cell $H^{\prime}$ of $D^{\prime}$ with top $t$ such that $D$ is obtained from $D^{\prime}$ by a (single) fork extension at $H^{\prime}$. By the minimality of $|D|, D^{\prime}$ is obtained from a grid by a sequence of multi-fork extensions at distributive 4-cells. If $H^{\prime}$ was a distributive 4-cell of $D^{\prime}$, then $D$ would not be a counterexample since the above-mentioned single fork extension is also a multi-fork extension.

Hence, $H^{\prime}$ is a non-distributive 4-cell of $D^{\prime}$. By the minimality of $|D|$, $D^{\prime}$ is obtained from a grid by multi-fork extensions at distributive 4-cells 
$H_{0}, \ldots, H_{k-1}$ of rectangular diagrams $D_{0}, \ldots, D_{k-1}$, respectively, where $D_{0}$ is a grid. We also denote $D^{\prime}$ and $D$ by $D_{k}$ and $D_{k+1}$, respectively. Let $t_{i} \in D_{i}$ denote the top $1_{H_{i}}$ of $H_{i}$ for $i \in\{0, \ldots, k-1\}$. Obviously (or by [12, Lemma $15]), \downarrow t_{i}$ is not distributive in $D_{j}$ for $j>i$. In particular, it is not distributive in $D$. The choice of $t$ implies that

$$
t_{i} \nless t \text { for } i=0, \ldots, k-1 \text {. }
$$

On the other hand, the non-distributivity of $\downarrow t$ in $D^{\prime}$ implies that $\downarrow t$ contains some cover-preserving $S_{7}$ sublattices of $D^{\prime}$. It is clear from definitions that the only cover-preserving $\mathrm{S}_{7}$ sublattice the $i$-th multi-fork extension creates contains $t_{i-1}$ as its largest element, and the $i$-th multi-fork extension does not change the tops of the previous $S_{7}$ 's. Therefore, there exists a $j \in\{0, \ldots, k-1\}$ such that $t_{j} \leq t$. That is, by $(3.1), t_{j}=t$. This $j$ is unique since the corresponding extension, which is the $(j+1)$-th, destroys the distributivity of any 4 -cells with top $t=t_{j}$. By the same reason, $t_{i} \ngtr t=t_{j}$ holds for all $i>j$. Combining this with (3.1), we obtain that $t_{j} \| t_{i}$ for $i \in\{j+1, \ldots, k-1\}$. Hence, Lemma 3.4 allows us to assume that $j=k-1$. But now Lemma 3.6 implies that $D$ is a multi-fork extension of $D_{k-1}$ at its distributive 4-cell $H_{k-1}$, which contradicts the assumption that $D$ is a counterexample.

The patch extention preserves slimness and semimodularity even if the 4cell in question is not distributive, see Proposition 3.3. Theorem 3.7 points out that it is at distributive 4-cells where multi-fork extensions are most important for slim rectangular lattices.

\section{Trajectory quasi-colorings}

The purpose of this section is turn the suggestion of Remark 2.1 into reality.

4.1. Quasi-colorings. Quasiordered sets, also called preordered sets, are relational structures $\langle A ; \nu\rangle$ such that $\nu \subseteq A^{2}$ is a quasiordering, that is, a reflexive, transitive relation. Quite often, especially if we intend to use the transitivity of $\nu$, we write $a \leq_{\nu} b$ or $b \geq_{\nu} a$ for $\langle a, b\rangle \in \nu$. We recall some basic properties from G. Grätzer [17]. Let $\nu^{n}$ denote $\nu \cap \nu^{-1}$, the equivalence induced by $\nu$. The ordering and the ordered set associated with the quasiordering $\nu$ and the quasiordered set $\langle A ; \nu\rangle$ are

$$
\nu^{\bullet}=\left\{\left(a / \nu^{n}, a / \nu^{n}\right):\langle a, b\rangle \in \nu\right\} \text { and }\left\langle A / \nu^{n} ; \nu^{\bullet}\right\rangle,
$$

respectively. This ordered set is used if we want to depict the quasi-ordered set $\langle A ; \nu\rangle$ : we draw the diagram of $\left(A / \nu^{n} ; \nu^{\bullet}\right)$, and label its elements by the

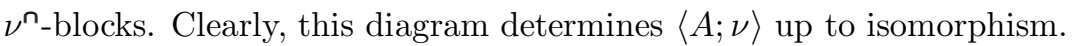

For $X \subseteq A^{2}$, the least quasiordering of $A$ that includes $X$ will be denoted by quor $_{A}(X)$, or simply by quor $(X)$ if there is no danger of confusion. We will, of course, write quor $(a, b)$ for quor $(\{\langle a, b\rangle\})$. The set of all quasiorderings on $A$ form a complete lattice Quo $A$ under set inclusion. For $\nu, \tau \in$ Quo $A$, the 
join $\nu \vee \tau$ is quor $(\nu \cup \tau)$. Note that the congruence generated by $X$ and that generated by $\{\langle a, b\rangle\}$ are denoted by $\operatorname{con}(X)$ and $\operatorname{con}(a, b)$, respectively, and $\operatorname{con}(a, b)=$ quor $\{\langle a, b\rangle,\langle b, a\rangle\}$.

Following G. Czédli [3], a quasi-colored lattice is a lattice $M$ of finite length with a surjective map $\gamma$, called quasi-coloring, from $\operatorname{PrInt}(M)$ onto a quasiordered set $(A ; \nu)$ such that $\gamma$ satisfies the following two properties:

(C1) if $\gamma(\mathfrak{p}) \geq_{\nu} \gamma(\mathfrak{q})$, then $\operatorname{con}(\mathfrak{p}) \geq \operatorname{con}(\mathfrak{q})$,

$(\mathrm{C} 2)$ if $\operatorname{con}(\mathfrak{p}) \geq \operatorname{con}(\mathfrak{q})$, then $\gamma(\mathfrak{p}) \geq_{\nu} \gamma(\mathfrak{q})$.

The importance of quasi-colorings is clear by the following lemma, which follows from G. Czédli [3, (2.6) and (2.7)]; note, however, that the lemma is an easy translation of its counterpart in G. Grätzer and E. Knapp [23], where it is attributed to J. Jakubík [27]. If $\mathfrak{p}=[u, v]$, then we write $\gamma(u, v)$ rather than $\gamma([u, v])$ or $\gamma(\langle u, v\rangle)$. The congruence lattice of a lattice $L$ is denoted by Con $L$.

Lemma 4.1. Let $K$ be a finite distributive lattice, and let $L$ be a finite lattice. Then $K \cong$ Con $L$ iff there exists a quasi-coloring $\gamma$ from $\operatorname{PrInt}(L)$ onto a quasiordered set $\langle A ; \nu\rangle$ such that the ordered set $\left\langle A / \nu^{\cap} ; \nu^{\bullet}\right\rangle$ associated with $\langle A ; \nu\rangle$ is isomorphic to $\langle$ Ji $K ; \leq\rangle$.

In the particular case where $\nu$ is an ordering, quasi-colorings are the traditional colorings introduced by G. Grätzer and E. Knapp [23]. The name "coloring" was used for surjective maps onto antichains satisfying $(\mathrm{C} 2)$ in G. Grätzer, H. Lakser, and E. T. Schmidt [25], and for surjective maps onto antichains satisfying (C1) in G. Grätzer [16, page 39]. Since Lemma 4.1 is also true and valuable if only colorings are considered, one may ask the question: Why trouble ourselves with quasi-colorings?

Remark 4.2. The first answer to this question is given in G. Czédli [3] as follows: since we have joins in Quo A, quasi-colorings give insight into complicated constructions by decomposing them into "elementary" steps and forming the "join" of the corresponding quasi-colorings. The second answer will be soon given in Theorem 4.4 and Remark 5.7; the point is that a quasi-coloring can be defined, illustrated, and treated easier than a coloring. The simplest quasicoloring, the identity map, occurs already in G. Grätzer [17, Theorem 239].

The key definition of the paper, which we give below, is a lattice concept, so it could be phrased for lattices instead of a diagrams. For its motivation, take the hat-trajectory $u$ containing $\left[s_{1}, t\right]$ and the up-trajectory $v$ containing $\left[w_{r}, t\right]$ of $S_{7}$ in Figure 1. Observe that our definition describes a straightforward reason for the inequality $\operatorname{con}\left(\left\langle s_{1}, t\right\rangle\right) \leq \operatorname{con}\left(\left\langle w_{r}, t\right\rangle\right)$.

Definition 4.3. Let $D$ be a slim semimodular lattice diagram.

(i) For a trajectory $u$ of $D$, the top edge $\mathfrak{h}=\mathfrak{h}(u)$ of $u$ is defined by the property $\mathfrak{h} \in u$ and $1_{\mathfrak{h}}>1_{\mathfrak{p}}$ holds for all $\mathfrak{p} \in u$. 
(ii) On the set $\operatorname{Traj}(D)$ of all trajectories of $D$, we define a relation $\sigma$ as follows. For $u, v \in \operatorname{Traj}(D)$, we let $u \leq_{\sigma} v$ iff $u$ is a hat-trajectory, $1_{\mathfrak{h}(u)} \leq 1_{\mathfrak{h}(v)}$, but $0_{\mathfrak{h}(u)} \not \leq 0_{\mathfrak{h}(v)}$.

(iii) We let $\tau=$ quor $(\sigma)$, the reflexive transitive closure of $\sigma$ on $\operatorname{Traj}(D)$.

(iv) The trajectory quasi-coloring of $D$ is the quasi-coloring $\xi$ from $\operatorname{Pr} \operatorname{Int}(D)$ onto $\langle\operatorname{Traj}(D), \tau\rangle$, defined by the rule $\mathfrak{p} \in \xi(\mathfrak{p})$.

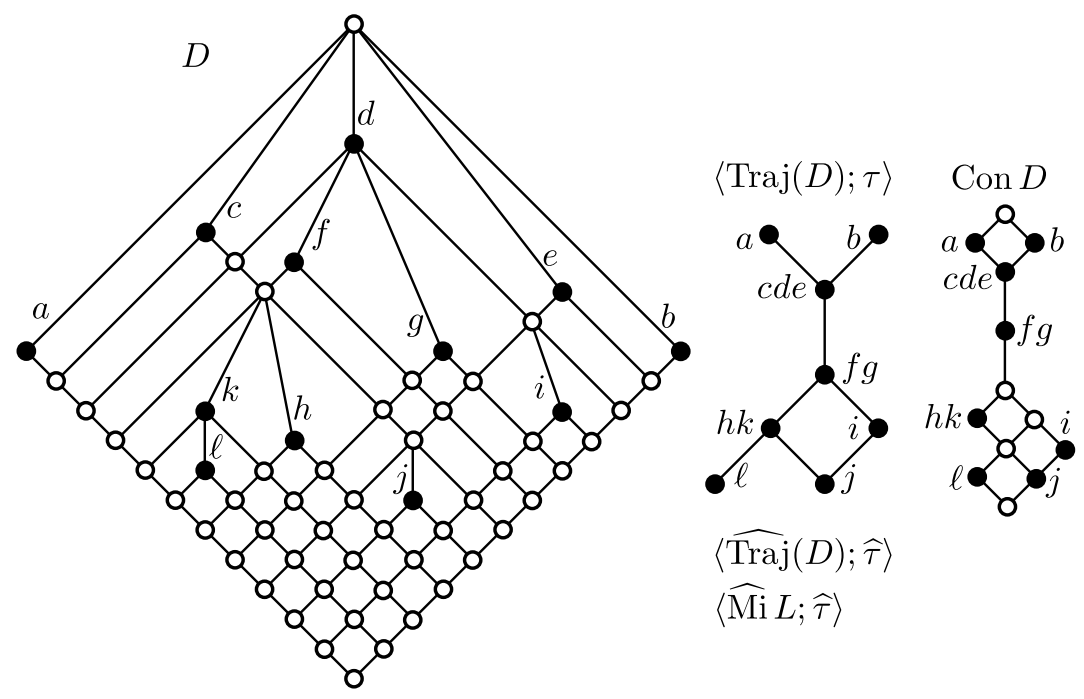

Figure 4. Illustration for Theorems 4.4 and 7.3

The values of $\xi$ will be called "colors" rather than "quasi-colors". While the existence of $\mathfrak{h}(u)$ above follows from (2.1), we have to prove that $\xi$ is a quasicoloring. Hence, we state the following proposition, but only for rectangular lattice diagrams.

Theorem 4.4 (Trajectory quasi-coloring theorem). If $D$ is a slim rectangular lattice diagram, then the map $\xi$ defined in Definition 4.3 is a quasi-coloring.

We illustrate Theorem 4.4 with the slim rectangular diagram $D$ depicted in Figure 4 . In the diagram, sets are written in short forms; for instance, cde denotes $\{c, d, e\}$. We have that $\operatorname{Traj}(D)=\{a, b, \ldots, k, \ell\}$, and these trajectories are labeled at their top edges. (The two lower right labels in the figure will be defined in Section 7.)

5. The properties of multi-fork extensions and the proof of Theorem 4.4

The proof of Theorem 4.4 needs several auxiliary statements. We will rely on Lemma 2.2 without referencing it. 


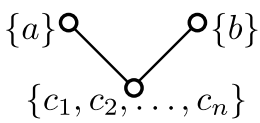

FIGURE 5. The diagram of $\left\langle\operatorname{Traj}\left(\mathrm{S}_{7}^{(n)}\right) ; \tau\right\rangle$

Lemma 5.1. For every $n \in \mathbb{N},\left\langle\operatorname{Traj}\left(\mathrm{S}_{7}^{(n)}\right) ; \tau\right\rangle$ is the quasiordered set given by Figure 5, which uses the notation of Figure 1. Furthermore, the trajectory quasi-coloring $\xi: \operatorname{PrInt}\left(\mathrm{S}_{7}^{(n)}\right) \rightarrow\left\langle\operatorname{Traj}\left(\mathrm{S}_{7}^{(n)}\right) ; \tau\right\rangle$ is a quasi-coloring.

Proof. For $n=1$, the statement is obvious. Hence, with the notation of Figure 1, the leftmost cover-preserving $\mathrm{S}_{7}$ sublattice gives that $\operatorname{con}\left(s_{1}, t\right) \leq$ $\operatorname{con}\left(s_{2}, t\right)$ if $n \geq 2$, while the next $\mathrm{S}_{7}$ sublattice to the right yields that $\operatorname{con}\left(s_{1}, t\right) \geq \operatorname{con}\left(s_{2}, t\right)$. Thus $\operatorname{con}\left(s_{1}, t\right)=\operatorname{con}\left(s_{2}, t\right)$. Similarly, $\operatorname{con}\left(s_{i}, t\right)=$ $\operatorname{con}\left(s_{i+1}, t\right)$ for all $i \leq n$. Since the equivalence with blocks $\{0\},\left[w_{\ell}, w_{\ell} \wedge s_{n}\right]$, $\left[s_{1} \wedge w_{r}, w_{r}\right]$, and $\left[s_{i} \wedge s_{n}, t\right]$ is a congruence, the rest of the lemma is obvious.

In the following lemma, we use the notation given in Definition 3.1. The relations "on the left" and "on the right" below are reflexive.

Lemma 5.2. Let $n \in \mathbb{N}$, and let $D$ be a slim semimodular diagram. If $H$ is a distributive 4-cell of $D$, then each $x \in D\left[S_{7}^{(n)} \leadsto H\right] \backslash D$ can uniquely be written into exactly one of the following forms (with unique $i \in\{1, \ldots, n\}$ and $v \in D$ ):

(i) $x=v \wedge s_{i}$, where $[u, v] \in \operatorname{PrInt}(D),[u, v] \stackrel{\text { traj }}{ }\left[0_{H}, \operatorname{lc}(H)\right]$ in $D$, and $[u, v]$ is on the left of $\left[0_{H}, \operatorname{lc}(H)\right]$ in the trajectory of $D$ through $\left[0_{H}, \operatorname{lc}(H)\right]$.

(ii) $x=v \wedge s_{i}$, where $[u, v] \in \operatorname{PrInt}(D),[u, v] \sim^{\operatorname{traj}}\left[0_{H}, \operatorname{rc}(H)\right]$ in $D$, and $[u, v]$ is on the right of $\left[0_{H}, \mathrm{rc}(H)\right]$ in the trajectory of $D$ through $\left[0_{H}, \mathrm{rc}(H)\right]$. (iii) $x$ is in the interior of $\mathrm{S}_{7}^{(n)}$.

Proof. Clearly, each element of $\mathrm{C}_{\ell \ell}\left(\mathrm{S}_{7}^{(n)}\right) \backslash\left\{0, \operatorname{lc}\left(\mathrm{S}_{7}^{(n)}\right)\right\}$ is of the unique form $\operatorname{lc}\left(\mathrm{S}_{7}^{(n)}\right) \wedge s_{i}$, see Figure 1, and analogously for the lower right boundary. Hence, the statement is an evident consequence of definitions, see also Figure 2.

Next, we formulate an important auxiliary statement.

Lemma 5.3 (Retraction lemma). Let $H$ be a distributive 4-cell of a slim semimodular lattice diagram $D$, and let $n \in \mathbb{N}$. Consider the retraction map $\psi: D\left[\mathrm{~S}_{7}^{(n)} \sim H\right] \rightarrow D$, defined by

$$
x \mapsto \begin{cases}x, & \text { if } x \in D, \\ v, & \text { if } x \text { belongs to the scope of Lemma 5.2(i), } \\ v, & \text { if } x \text { belongs to the scope of Lemma 5.2(ii), } \\ 1_{H}, & \text { if } x \text { belongs to the scope of Lemma 5.2(iii). }\end{cases}
$$

Then $\psi$ is a lattice homomorphism. 
Proof. Let $D^{\prime}$ denote $D\left[\mathrm{~S}_{7}^{(n)} \leadsto H\right]$. First we show that $\boldsymbol{\alpha}:=\operatorname{Ker}(\psi)$ is a lattice congruence. The non-singleton $\boldsymbol{\alpha}$-blocks are the following:

$$
\begin{aligned}
E & =\left[s_{1} \wedge s_{n}, 1_{H}\right]=\left[s_{1} \wedge \cdots \wedge s_{n}, 1_{H}\right], \\
F_{v} & =\left[v \wedge s_{n}, v\right]=\left\{v, v \wedge s_{1}, \ldots, v \wedge s_{n}\right\} \text { for } v \text { from Lemma 5.2(i), } \\
G_{v} & =\left[v \wedge s_{1}, v\right]=\left\{v, v \wedge s_{1}, \ldots, v \wedge s_{n}\right\} \text { for } v \text { from Lemma 5.2(ii). }
\end{aligned}
$$

In Figure 2, these $\boldsymbol{\alpha}$-blocks are indicated by dotted closed curves. We know from G. Czédli and E.T. Schmidt [12, Lemma 2] that every element in a slim lattice has at most two covers. Hence, the condition on upper covers in Lemma 2.2 follows easily from (5.1). On the other hand, $\downarrow 1_{H}$ is clearly a planar lattice, and it is distributive by the assumption on $H$. Planar distributive lattices are always slim and dually slim by G. Czédli and E. T. Schmidt [12, Lemma 16] and G. Grätzer and E. Knapp [21]. Hence, understanding $\downarrow 1_{H}$ in $D$, we have that

$$
\text { each } \left.x \in \downarrow 1_{H} \text { has at most two lower covers in } D\right) \text {. }
$$

Therefore, an element in one of the non-singleton $\boldsymbol{\alpha}$-blocks (5.1) has only those lower covers that are depicted in Figure 2. Hence, it is straightforward to see that $\boldsymbol{\alpha}$ satisfies the condition on lower covers in Lemma 2.2. Thus we conclude that $\boldsymbol{\alpha}$ is a lattice congruence on $D^{\prime}$.

Since $\psi$ is idempotent,

$$
\langle z, \psi(z)\rangle \in \operatorname{Ker} \psi=\boldsymbol{\alpha} \text { for all } z \in D^{\prime} .
$$

Let $x, y \in D^{\prime}$. Since $\langle x, \psi(x)\rangle$ and $\langle y, \psi(y)\rangle$ belong to $\boldsymbol{\alpha}$ by (5.3), we obtain that $\langle x \vee y, \psi(x) \vee \psi(y)\rangle \in \boldsymbol{\alpha}$. But $\langle\psi(x \vee y), x \vee y\rangle$ by (5.3), and transitivity yields that

$$
\langle\psi(x \vee y), \psi(x) \vee \psi(y)\rangle \in \boldsymbol{\alpha} .
$$

Clearly, both $\psi(x \vee y)$ and $\psi(x) \vee \psi(y)$ belong to $D$ since $\psi$-images are in $D$ and $D$ is a sublattice. The description (5.1) of $\boldsymbol{\alpha}$-blocks makes it clear that each $\boldsymbol{\alpha}$-block intersects $D$ in a singleton. Hence, (5.4) implies that $\psi(x \vee y)=$ $\psi(x) \vee \psi(y)$. This proves that $\psi$ is a join-homomorphism. It follows similarly that it is also a meet-homomorphism.

Definition 5.4. Let $H$ be a distributive 4-cell of a slim semimodular lattice diagram $D$, and let $n \in \mathbb{N}$. Let $\gamma: \operatorname{PrInt}(D) \rightarrow\langle A ; \nu\rangle$ be a quasi-coloring, and let $\xi: \operatorname{PrInt}\left(\mathrm{S}_{7}^{(n)}\right) \rightarrow\left\langle\operatorname{Traj}\left(\mathrm{S}_{7}^{(n)}\right) ; \tau\right\rangle$ be the trajectory quasi-coloring of $\mathrm{S}_{7}^{(n)}$, described by Lemma 5.1. We also write $B=\operatorname{Traj}\left(\mathrm{S}_{7}^{(n)}\right)$ and $D^{\prime}=D\left[\mathrm{~S}_{7}^{(n)} \sim H\right]$. Assume also that

$$
\begin{aligned}
& \gamma\left(\operatorname{lc}(H), 1_{H}\right)=a=\xi\left(\operatorname{lc}\left(\mathrm{S}_{7}^{(n)}\right), 1_{\left.\mathrm{S}_{7}^{(n)}\right)}\right), \\
& \gamma\left(\operatorname{rc}(H), 1_{H}\right)=b=\xi\left(\operatorname{rc}\left(\mathrm{S}_{7}^{(n)}\right), 1_{\mathrm{S}_{7}^{(n)}}\right), \text { and } A \cap B=\{a, b\} .
\end{aligned}
$$


On the set $C=A \cup B$, we define $\eta=\operatorname{quor}(\nu \cup \tau)$. Also, we define a map $\delta: \operatorname{PrInt}\left(D\left[\mathrm{~S}_{7}^{(n)} \sim H\right]\right) \rightarrow\langle C ; \eta\rangle$ by

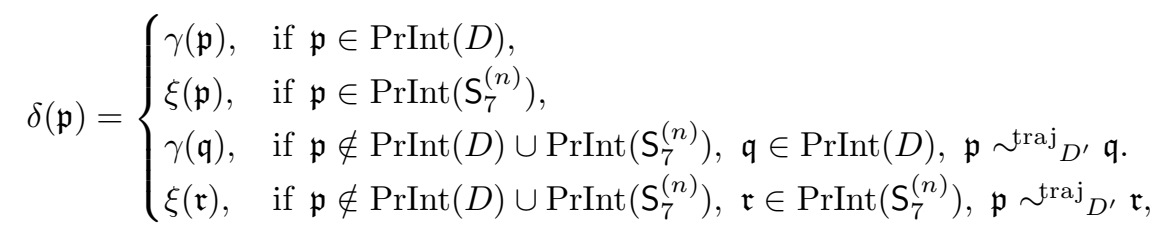

where we also stipulate that $\mathfrak{q}$ is the edge of the trajectory of $\mathfrak{p}$ nearest to $\mathfrak{p}$ such that $1_{\mathfrak{q}} \geq 1_{\mathfrak{p}}$. (The distance of two edges in a trajectory of $D\left[\mathrm{~S}_{7}^{(n)} \leadsto H\right]$ is measured by the number of 4-cells of the trajectory between the two edges.) Note that $\mathfrak{q}$ is of the form $\left[v \wedge s_{i}, v^{\prime} \wedge s_{i}\right]$, where $v \prec v^{\prime}$ and either we have that $[u, v] \stackrel{\sim}{\mathrm{traj}}_{D}\left[u^{\prime}, v^{\prime}\right] \stackrel{\mathrm{traj}}{D}^{\mathrm{ta}}\left[0_{H}, \mathrm{lc}(H)\right]$ according to Lemma 5.2(i), or we have that $[u, v] \sim^{\text {traj }} D\left[u^{\prime}, v^{\prime}\right] \sim^{\mathrm{traj}_{D}}\left[0_{H}, \mathrm{rc}(H)\right]$ according to $5.2(\mathrm{ii})$. As opposed to $\mathfrak{q}$, the prime interval $\mathfrak{r}$ above is not unique. However, $\xi(\mathfrak{r})$ is unique, because $\xi$ is the trajectory quasi-coloring on $S_{7}^{(n)}$. Note also that, with the same notation as above, $\mathfrak{r}$ can always be chosen either as $\left[v \wedge s_{i}, v \wedge s_{i-1}\right]$ for some $i \in$ $\{1, \ldots, n+1\}$, according to $5.2(\mathrm{i})$, or as $\left[v \wedge s_{i}, v \wedge s_{i+1}\right]$ for some $i \in\{0, \ldots, n\}$, according to 5.2(ii). Finally, we note that if both $\mathfrak{q}$ and $\mathfrak{r}$ above exist, then (5.5) implies that they do not conflict and $\delta(\mathfrak{p}) \in\{a, b\}$.

Besides serving as an auxiliary statement in the proof of Theorem 4.4, the following theorem can be useful to construct slim semimodular lattices with given congruence lattices.

Theorem 5.5 (Multi-fork theorem). With the assumptions of Definition 5.4, $\delta$ is a quasi-coloring.

Corollary 5.6. If the 4-cell in question is distributive, then the (single) fork lemma (that is, [3, Lemma 5.1]) holds.

Remark 5.7. Although the stipulation (5.5) seems to hold rarely, this is not a real obstacle to the applicability of Theorem 5.5. First, because if we have that $\gamma\left(\operatorname{lc}(H), 1_{H}\right) \neq \gamma\left(\operatorname{rc}(H), 1_{H}\right)$, then (5.5) will hold after renaming the $\gamma$-colors. Second, if we have that $\gamma\left(\operatorname{lc}(H), 1_{H}\right)=\gamma\left(\operatorname{rc}(H), 1_{H}\right)=a$, then we can modify $\gamma$ by adding a new color $a^{\prime}$ to $A$, replacing $\nu$ by $\nu^{\prime}=$ quor $\left(\nu \cup\left\{\left\langle a, a^{\prime}\right\rangle,\left\langle a^{\prime}, a\right\rangle\right\}\right)$, and changing $\gamma\left(\operatorname{rc}(H), 1_{H}\right)$ to $a^{\prime}$; after these changes, the previous case applies. As an argument for quasi-colorings, note that we could not take $\nu^{\prime}$ if we dealt with colorings rather than quasi-colorings.

Proof of Theorem 5.5. To show that $\delta$ satisfies (C1), we assume that $\mathfrak{p}, \mathfrak{q} \in$ $\operatorname{PrInt}\left(D^{\prime}\right)$ such that $\delta(\mathfrak{p}) \geq_{\eta} \delta(\mathfrak{q})$. We have a sequence $\delta(\mathfrak{p})=a_{0}, a_{1}, \ldots, a_{k}=$ $\delta(\mathfrak{q})$ in $C$ such that $\left\langle a_{i-1}, a_{i}\right\rangle \in \nu \cup \tau$ for $i \in\{1, \ldots, k\}$. (Note that if $\delta(\mathfrak{p})=$ $\delta(\mathfrak{q})$, then we can let $k=1$ since $\nu \cup \tau$ is reflexive.) Clearly, $\delta$ is surjective. Moreover, even its restriction to $\operatorname{PrInt}(D) \cup \operatorname{PrInt}\left(\mathrm{S}_{7}^{(n)}\right)$ is surjective. Hence, we can pick $\mathfrak{r}_{i} \in \operatorname{PrInt}(D) \cup \operatorname{PrInt}\left(\mathrm{S}_{7}^{(n)}\right)$ such that $a_{i}=\delta\left(\mathfrak{r}_{i}\right)$ for $i \in\{1, \ldots, k\}$. 
For $\mathfrak{r}, \mathfrak{r}^{\prime} \in \operatorname{PrInt}\left(D^{\prime}\right)$, the inclusion $\operatorname{con}_{D^{\prime}}(\mathfrak{r}) \supseteq \operatorname{con}_{D^{\prime}}\left(\mathfrak{r}^{\prime}\right)$ holds iff $\operatorname{con}_{D^{\prime}}(\mathfrak{r})$ collapses $\mathfrak{r}^{\prime}$. Using (2.3) and the fact that $D$ and $\mathrm{S}_{7}^{(n)}$ are sublattices of $D^{\prime}$, it follows easily that if $\mathfrak{r}, \mathfrak{r}^{\prime} \in \operatorname{PrInt}(D)$ or $\mathfrak{r}, \mathfrak{r}^{\prime} \in \operatorname{PrInt}\left(\mathrm{S}_{7}^{(n)}\right)$, then $\operatorname{con}_{D}(\mathfrak{r}) \supseteq$ $\operatorname{con}_{D}\left(\mathfrak{r}^{\prime}\right)$ or $\operatorname{con}_{\mathrm{S}_{7}^{(n)}}(\mathfrak{r}) \supseteq \operatorname{con}_{\mathrm{S}_{7}^{(n)}}\left(\mathfrak{r}^{\prime}\right)$ implies that $\operatorname{con}_{D^{\prime}(\mathfrak{r})} \supseteq \operatorname{con}_{D^{\prime}}\left(\mathfrak{r}^{\prime}\right)$. Thus, since both $\gamma$ and $\xi$ are quasi-colorings and $\delta$ extends them, the containment $\left\langle\delta\left(\mathfrak{r}_{i-1}\right), \delta\left(\mathfrak{r}_{i}\right)\right\rangle=\left\langle a_{i-1}, a_{i}\right\rangle \in \nu \cup \tau$ implies that $\operatorname{con}_{D^{\prime}}\left(\mathfrak{r}_{i-1}\right) \supseteq \operatorname{con}_{D^{\prime}}\left(\mathfrak{r}_{i}\right)$ for $i \in\{1, \ldots, k\}$. Hence, transitivity yields that $\operatorname{con}_{D^{\prime}}(\mathfrak{p}) \supseteq \operatorname{con}_{D^{\prime}}(\mathfrak{q})$, proving that $\delta$ satisfies $(\mathrm{C} 1)$.

Next, to show that $\delta$ satisfies (C2), we assume that $\mathfrak{p}_{1}, \mathfrak{p}_{2} \in \operatorname{PrInt}\left(D^{\prime}\right)$ such that $\operatorname{con}_{D^{\prime}}\left(\mathfrak{p}_{1}\right) \supseteq \operatorname{con}_{D^{\prime}}\left(\mathfrak{p}_{2}\right)$. We want to show that $\delta\left(\mathfrak{p}_{1}\right) \geq_{\eta} \delta\left(\mathfrak{p}_{2}\right)$. We have to deal with three cases.

Case 1. We assume that $\left\{\delta\left(\mathfrak{p}_{1}\right), \delta\left(\mathfrak{p}_{2}\right)\right\} \subseteq A$. By $(2.3), \mathfrak{p}_{1} \Rightarrow D_{D^{\prime}} \mathfrak{p}_{2}$. Hence, there are intervals $\mathfrak{r}_{i}=\left[x_{i}, y_{i}\right] \in \operatorname{Int}\left(D^{\prime}\right)$ that form a sequence

$$
\mathfrak{p}_{1}=\mathfrak{r}_{0} \rightarrow D^{\prime} \mathfrak{r}_{1} \rightarrow D^{\prime} \cdots \rightarrow D^{\prime} \mathfrak{r}_{k}=\mathfrak{p}_{2} .
$$

Note that $\{a, b\} \cap\left\{c_{1}, \ldots, c_{n}\right\}=\varnothing$ by Lemma 5.1. Hence, $\left\{c_{1}, \ldots, c_{n}\right\} \cap A=\varnothing$ by (5.5). Observe that if a prime interval $\mathfrak{q} \in \operatorname{PrInt}\left(D^{\prime}\right)$ is collapsed by the retraction homomorphism $\psi$ from Lemma 5.3, then its $\delta$-color is one of the $c_{i}$, $i \in\{1, \ldots, n\}$. Therefore, we conclude that none of $\mathfrak{p}_{1}$ and $\mathfrak{p}_{2}$ is collapsed by $\psi$. The map $\psi$ sends (5.6) to a congruence-perspectivity sequence

$$
\psi\left(\mathfrak{p}_{1}\right)=\psi\left(\mathfrak{r}_{0}\right) \rightarrow_{D} \psi\left(\mathfrak{r}_{1}\right) \rightarrow_{D} \cdots \rightarrow_{D} \psi\left(\mathfrak{r}_{k}\right)=\psi\left(\mathfrak{p}_{2}\right) ;
$$

however, we have to verify that the $\psi\left(\mathfrak{r}_{i}\right)$ are nontrivial intervals. If one of the $\mathfrak{r}_{i}$ was collapsed by $\psi$, then the defining relations of $\rightarrow$, together with (5.7) and (2.3), would imply that $\operatorname{con}_{D}\left(\psi\left(\mathfrak{r}_{i}\right)\right) \supseteq \operatorname{con}_{D}\left(\psi\left(\mathfrak{p}_{2}\right)\right)$. This would be a contradiction, because then the equality relation would collapse $\psi\left(\mathfrak{p}_{2}\right)$, which is a nontrivial interval since $\psi$ does not collapse $\mathfrak{p}_{2}$. Thus none of the $\mathfrak{r}_{i}$ is collapsed by $\psi$. That is, the $\psi\left(\mathfrak{r}_{i}\right)$ are nontrivial intervals, as claimed. Using (2.3) again, we obtain that $\operatorname{con}_{D}\left(\psi\left(\mathfrak{p}_{1}\right)\right) \supseteq \operatorname{con}_{D}\left(\psi\left(\mathfrak{p}_{2}\right)\right)$. Since $\gamma$ is a quasi-coloring, we conclude that $\gamma\left(\psi\left(\mathfrak{p}_{1}\right)\right) \geq_{\nu} \gamma\left(\psi\left(\mathfrak{p}_{2}\right)\right)$. This implies that $\gamma\left(\psi\left(\mathfrak{p}_{1}\right)\right) \geq_{\eta} \gamma\left(\psi\left(\mathfrak{p}_{2}\right)\right)$. It follows from the definitions that,

$$
\delta(\mathfrak{p}) \in A \Longrightarrow \delta(\mathfrak{p})=\gamma(\psi(\mathfrak{p}))
$$

for every $\mathfrak{p} \in \operatorname{PrInt}\left(D^{\prime}\right)$. Thus we obtain $\delta\left(\mathfrak{p}_{1}\right) \geq_{\eta} \delta\left(\mathfrak{p}_{2}\right)$, completing Case 1 .

Case 2. We assume that $\delta\left(\mathfrak{p}_{1}\right) \notin A$. This means that $\delta\left(\mathfrak{p}_{1}\right)=c_{i}$ for some $i \in\{1, \ldots, n\}$. Clearly,

$$
\mathfrak{p}_{1} \text { is congruence-equivalent to }\left[s_{i}, 1_{H}\right]
$$

since they belong to the same trajectory. Let $\boldsymbol{\alpha}$ denote Ker $\psi$ from Lemma 5.3, see Figure 2. Clearly, $\operatorname{con}_{D^{\prime}}\left(\mathfrak{p}_{1}\right) \subseteq \boldsymbol{\alpha}$. It follows from Lemma 5.1 that the restriction $\boldsymbol{\alpha}]_{S_{7}^{(n)}}$ of $\boldsymbol{\alpha}$ to $S_{7}^{(n)}$ is an atom in $\operatorname{Con}_{7}^{(n)}$. Therefore, Figure 2 shows that $\boldsymbol{\alpha}$ is an atom in Con $D^{\prime}$. Thus $\operatorname{con}_{D^{\prime}}\left(\mathfrak{p}_{1}\right)=\boldsymbol{\alpha}$. Hence, the assumption $\operatorname{con}_{D^{\prime}}\left(\mathfrak{p}_{1}\right) \supseteq \operatorname{con}_{D^{\prime}}\left(\mathfrak{p}_{2}\right)$ implies that $\mathfrak{p}_{2}$ lies in an $\boldsymbol{\alpha}$-class. By definition, we 
obtain that $\delta\left(\mathfrak{p}_{2}\right)=c_{j}$ for some $j \in\{1, \ldots, n\}$. Since $c_{i} \geq_{\tau} c_{j}$ by Lemma 5.1, we conclude that $\delta\left(\mathfrak{p}_{1}\right)=c_{i} \geq_{\eta} c_{j}=\delta\left(\mathfrak{p}_{2}\right)$, as claimed.

Case 3. We assume that $\delta\left(\mathfrak{p}_{1}\right) \in A$ but $\delta\left(\mathfrak{p}_{2}\right) \notin A$. We want to show that $\delta\left(\mathfrak{p}_{1}\right) \geq_{\eta} \delta\left(\mathfrak{p}_{2}\right)$. By (5.8) and (with change of the subscript) (5.9), we can also assume that $\mathfrak{p}_{1} \in \operatorname{PrInt}(D), \mathfrak{p}_{2} \in \operatorname{PrInt}\left(\mathrm{S}_{7}^{(n)}\right)$, the top of $\mathfrak{p}_{2}$ is $1_{H}$, and its bottom is in $\left\{s_{1}, \ldots, s_{n}\right\}$, see Figure 2. Hence, $\delta\left(\mathfrak{p}_{2}\right) \in\left\{c_{1}, \ldots, c_{n}\right\}$. Temporarily, we adopt the following terminology. An interval $[x, y]$ is old, if $x, y \in D$. (Note, however, that an old interval, such as $[0,1]$, can contain new elements, that is, elements from $\mathrm{S}_{7}^{(n)} \backslash D$.) If $\{x, y\} \cap D=\varnothing$, then $[x, y]$ is new. The rest of the intervals are mixed. A mixed $[x, y]$ is an [old,new] interval if $x \in D$ and $y \notin D$, and it is a [new,old] interval if $x \notin D$ and $y \in D$. For example, $\mathfrak{p}_{2}$ is a [new,old] interval. If $x \leq x^{\prime} \leq y^{\prime} \leq y$, then $\left[x^{\prime}, y^{\prime}\right]$ is a subinterval of $[x, y]$. Observe that it suffices to show that

$$
\operatorname{con}_{D^{\prime}}\left(\mathfrak{p}_{1}\right) \text { collapses an [old,new] interval. }
$$

We can argue for (5.10) as follows. We easily obtain from definitions, see Figure 2, that for every [old,new] interval $[x, y], \operatorname{con}_{D^{\prime}}(x, y)$ contains $\left\langle\operatorname{lc}(H), 1_{H}\right\rangle=$ $\left\langle w_{\ell}, 1_{H}\right\rangle$ or $\left\langle\operatorname{rc}(H), 1_{H}\right\rangle=\left\langle w_{r}, 1_{H}\right\rangle$. Therefore, we have that $\operatorname{con}_{D^{\prime}}(x, y) \geq$ $\operatorname{con}_{D^{\prime}}\left(w_{\ell}, 1_{H}\right)$ or $\operatorname{con}_{D^{\prime}}(x, y) \geq \operatorname{con}_{D^{\prime}}\left(w_{r}, 1_{H}\right)$. Hence, (5.10) would imply that $\operatorname{con}_{D^{\prime}}\left(\mathfrak{p}_{1}\right) \geq \operatorname{con}_{D^{\prime}}\left(w_{\ell}, 1_{H}\right)$ or $\operatorname{con}_{D^{\prime}}\left(\mathfrak{p}_{1}\right) \geq \operatorname{con}_{D^{\prime}}\left(w_{\ell}, 1_{H}\right)$. Consequently, Case 1 yields that (5.10) would imply that $\delta\left(\mathfrak{p}_{1}\right) \geq_{\eta} \delta\left(w_{\ell}, 1_{H}\right)=a$ or $\delta\left(\mathfrak{p}_{1}\right) \geq_{\eta}$ $\delta\left(w_{r}, 1_{H}\right)=b$. Since $\delta\left(\mathfrak{p}_{2}\right) \in\left\{c_{1}, \ldots, c_{n}\right\}$ and we know that $a \geq_{\eta} c_{i}$ and $b \geq_{\eta} c_{i}$ for $i \in\{1, \ldots, n\}$, now it is clear by the transitivity of $\eta$ that (5.10) would imply the desired $\delta\left(\mathfrak{p}_{1}\right) \geq_{\eta} \delta\left(\mathfrak{p}_{2}\right)$. This verifies (5.10).

Observe that $\left[w_{\ell}, 1_{H}\right]$ is transposed to $\left[0_{H}, s_{1} \wedge w_{r}\right]$, which is an [old,new] interval. Similarly, $\left[w_{r}, 1_{H}\right]$ is transposed to the [old,new] interval $\left[0_{H}, s_{n} \wedge w_{\ell}\right]$. Hence, by (5.10), it suffices to show that

$$
\operatorname{con}_{D^{\prime}}\left(\mathfrak{p}_{1}\right) \text { collapses }\left[w_{\ell}, 1_{H}\right] \text { or }\left[w_{r}, 1_{H}\right] \text {. }
$$

By (2.3), there exists a sequence of intervals $\mathfrak{r}_{i}=\left[x_{i}, y_{i}\right] \in \operatorname{Int}\left(D^{\prime}\right)$ such that (5.6) holds. We assume that

the sequence (5.6) minimizes the number of new intervals

it contains. It suffices to show that there exists an $i \in\{0, \ldots, k\}$ such that

$\operatorname{con}_{D^{\prime}}\left(\mathfrak{r}_{i}\right)$ collapses or, in particular, contains an [old,new] interval,

since this would clearly imply (5.10). The sequence (5.6) begins with an old interval and terminates with a [new,old] one. Hence, there exists a smallest $i \in\{1, \ldots, k\}$ such that $\mathfrak{r}_{i}$ is not an old interval. In virtue of (5.13), there are only two subcases to consider.

Subcase $3 a$. We assume that $\mathfrak{r}_{i}$ is a new interval. We need the following terminology. A chain of new elements outside the interior of $\mathrm{S}_{7}^{(n)}$ is a parallel chain on the left if it is of the form $\left[v \wedge s_{i}, v^{\prime} \wedge s_{i}\right]$ where $[u, v]$ and $\left[u^{\prime}, v^{\prime}\right]$ belong to the trajectory of $D$ through $\left[0_{H}, w_{\ell}\right]$ and they are both on the left 

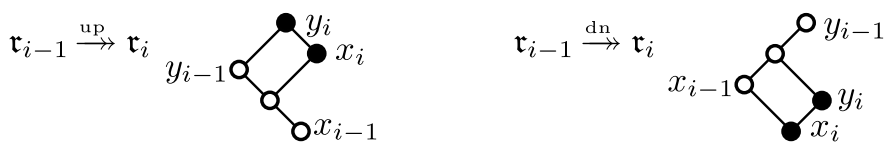

FiguRE $6 . \mathfrak{r}_{i-1} \rightarrow \mathfrak{r}_{i}$ in Subcase 3a

of $\left[0_{H}, w_{\ell}\right]$. Similarly, a parallel chain on the right is of the form $\left[v \wedge s_{i}, v^{\prime} \wedge s_{i}\right]$ where $[u, v]$ and $\left[u^{\prime}, v^{\prime}\right]$ belong to the trajectory of $D$ through $\left[0_{H}, w_{r}\right]$ and they are both on the right of $\left[0_{H}, w_{r}\right]$. If left or right is irrelevant, then the chain above is called a parallel chain. We claim that, unless another argument settles Subcase 3a,

$$
\mathfrak{r}_{i} \text { is a parallel chain. }
$$

To show this, assume first that $\mathfrak{r}_{i-1} \stackrel{\text { dn }}{\rightarrow} \mathfrak{r}_{i}$, see Figure 6 . Let $y_{i}$ belong to the interior of $\mathrm{S}_{7}^{(n)}$. Since the least element above $y_{i}$ in $D$ is $1_{H}$, we obtain $y_{i-1} \geq 1_{h}$. However, $x_{i-1} \nsucceq 1_{h}$ since otherwise $x_{i}=x_{i-1} \wedge y_{i}$ would equal $y_{i}$. Hence, $x_{i-1} \wedge 1_{H}<1_{H}=y_{i-1} \wedge 1_{H}$, and $\operatorname{con}_{D^{\prime}}\left(\mathfrak{r}_{i-1}\right)$ collapses the interval $\left[x_{i-1} \wedge 1_{H}, 1_{H}\right]$. We conclude from $(5.2)$ that $\left[w_{\ell}, 1_{H}\right]$ or $\left[w_{r}, 1_{H}\right]$ is a subinterval of $\left[x_{i-1} \wedge 1_{H}, 1_{H}\right]$. Hence, (5.11) settles the case where $y_{i}$ is in the interior of $\mathrm{S}_{7}^{(n)}$. Therefore, we can assume that $y_{i}$ is not in the interior of $\mathrm{S}_{7}^{(n)}$. By leftright symmetry, we can also assume that $y_{i}$ is on the left of $0_{H}$ or it belongs to $\left[0_{H}, w_{\ell}\right]$. That is, $y_{i}$ belongs to a cover-preserving $C_{2} \times C_{n+2}$ sublattice that we obtained from a 4-cell of $₫ A$ in Definition 3.1. Since $D$ is a sublattice of $D^{\prime}$ for every element $x \in D$, we use the following notation:

$$
x^{+}=D \cap \uparrow x, \quad x^{-}=D \cap \downarrow x .
$$

Note that $x \in D$ iff $x^{-}=x=x^{+}$. Returning to $y_{i}$, we have that $y_{i-1} \geq y_{i}^{+}$ and, since $x_{i-1} \wedge y_{i}=x_{i}<y_{i}$, we also have that $x_{i-1} \geq y_{i}^{+}$. Using $x_{i}<x_{i}^{+} \leq$ $x_{i-1}$, it follows that $x_{i}=x_{i}^{+} \wedge y_{i}$. By the definition of multi-fork extensions, see Figure 2, this clearly implies that $\mathfrak{r}_{i}$ is a parallel chain.

Next, we assume that $\mathfrak{r}_{i-1} \stackrel{\text { up }}{\rightarrow} \mathfrak{r}_{i}$, see Figure 6 . Assume that $x_{i} \in \uparrow 0_{H}$. Since $y_{i}$ is new element, it also belongs to the filter $\uparrow 0_{H}$. But $y_{i-1}$ is an old element, whence $y_{i-1} \leq y_{i}^{-}=x_{i}^{-}=0_{H}<x_{i}$. This contradicts that $y_{i-1} \vee x_{i}=y_{i}>x_{i}$. Thus, $x_{i} \notin \uparrow 0_{H}$. By left-right symmetry, we can assume that $x_{i}$ is on the right of $0_{H}$. It follows from $y_{i-1} \leq y_{i}^{-} \leq y_{i}$ and $y_{i}=y_{i-1} \vee x_{i}$ that $y_{i}=y_{i}^{-} \vee x_{i}$. Observe that $y_{i}$ is not in the interior of $\mathbf{S}_{7}^{(n)}$, because otherwise $y_{i}=y_{i}^{-} \vee x_{i}=0_{H} \vee x_{i}$, which is clearly not in the interior of $\mathrm{S}_{7}^{(n)}$. Hence, the construction yields that $\mathfrak{r}_{i}$ is a parallel chain. This completes the proof of (5.14).

We say that a parallel chain $\mathfrak{r}_{j}$ is on the left or on the right of $0_{H}$ depending on the position of $x_{j}$ with respect to $0_{H}$. Next, we prove that

if $\mathfrak{r}_{j}$ is a parallel chain, then either $\mathfrak{r}_{j+1}$ is an old interval, or it is a parallel chain on the same side of $0_{H}$ as $\mathfrak{r}_{j}$. 
To prove (5.16), assume that $\mathfrak{r}_{j+1}$ is not an old interval. First, let $\mathfrak{r}_{j} \stackrel{\text { up }}{\rightarrow} \mathfrak{r}_{j+1}$. By left-right symmetry (to harmonize with Figure 6), let $\mathfrak{r}_{j}$ be on the right. We can assume that $y_{j+1} \neq y_{j}$, that is, $x_{j+1} \| y_{j}$, since otherwise (5.16) clearly holds. If $x_{j+1}$ is a new element, then $x_{j} \leq x_{j+1} \| y_{j}$ yields that $x_{j+1}$ is on the right of $y_{j}$, we also have that $y_{j+1}=y_{j} \vee x_{j+1}$, and we clearly obtain that $\mathfrak{r}_{j+1}$ is a parallel chain on the same (right) side of $0_{H}$. Hence, we can assume that $x_{j+1}$ is an old element. Since $x_{j} \leq x_{j+1}$ gives that $x_{j}^{+} \leq x_{j+1}$, we obtain that $y_{j+1}=y_{j} \vee x_{j+1}=y_{j} \vee x_{j}^{+} \vee x_{j+1}=y_{j}^{+} \vee x_{j+1} \in D$, which contradicts the assumption that $\mathfrak{r}_{j+1}$ is not an old interval.

Second, let $\mathfrak{r}_{j} \stackrel{\text { dn }}{\rightarrow} \mathfrak{r}_{j+1}$. We can assume that $x_{j+1} \neq x_{j}$, that is, $y_{j+1} \| x_{j}$, since otherwise (5.16) clearly holds. By left-right symmetry, let $\mathfrak{r}_{j}$ be on the left. If $y_{j+1}$ is a new element, then $y_{j} \geq y_{j+1} \| x_{j}$ gives that $y_{j+1}$ is on the right $x_{j}$ but on the left of $0_{H}$, and $\mathfrak{r}_{j+1}$ is also a parallel chain on the left. Hence, we assume that $y_{j+1}$ is an old element. Since $y_{j} \geq y_{j+1}$ gives that $y_{j}^{-} \geq y_{j+1}$, we obtain that $x_{j+1}=x_{j} \wedge y_{j+1}=x_{j} \wedge y_{j}^{-} \wedge y_{j+1}=x_{j}^{-} \wedge y_{j+1} \in D$. This contradicts the assumption that $\mathfrak{r}_{j+1}$ is not an old interval, completing the proof of (5.16).

Now, we are in the position to complete Subcase 3a. We have assumed that $\mathfrak{r}_{i}$ is a new interval. Let $j$ be the smallest subscript such that $j \geq i$ and all the intervals in the subsequence

$$
\mathfrak{r}_{i} \rightarrow D^{\prime} \mathfrak{r}_{i+1} \rightarrow D^{\prime} \ldots \rightarrow D^{\prime} \mathfrak{r}_{j}
$$

are new but $\mathfrak{r}_{j+1}$ is not new. The existence of this $j$ (possibly $j=i$ ) follows from the fact that $\mathfrak{r}_{k}=\mathfrak{p}_{2}$ is not new. It follows from (5.14) and (5.16) that $\mathfrak{r}_{j+1}$ is an old interval. We obtain from (5.14) that, for every $m \in\{i, \ldots, j\}$, $\mathfrak{r}_{m}$ is an interval transposed to (and, therefore, congruence-equivalent to) both $\left[x_{m}^{-}, y_{m}^{-}\right]$and $\left[x_{m}^{+}, y_{m}^{+}\right]$. By (5.16), we can assume that, say, all these $\mathfrak{r}_{m}$ are on the left of $0_{H}$. Since both the maps $x \mapsto x^{-}$and $x \mapsto x^{+}$, defined on the set of new elements belonging to $\downarrow w_{\ell}$, are lattice homomorphisms,

$$
\left[x_{i}^{-}, y_{i}^{-}\right] \rightarrow D^{\prime} \ldots \rightarrow D^{\prime}\left[x_{j}^{-}, y_{j}^{-}\right] \text {and }\left[x_{i}^{+}, y_{i}^{+}\right] \rightarrow D^{\prime} \ldots \rightarrow D^{\prime}\left[x_{j}^{+}, y_{j}^{+}\right] .
$$

This implies easily that we can get rid of all the new intervals in (5.17) by replacing them with appropriate old intervals from (5.18), and adding one of the perspectivities

$$
\left[x_{i}^{-}, y_{i}^{-}\right] \stackrel{\text { up }}{\longrightarrow}\left[x_{i}^{+}, y_{i}^{+}\right], \quad\left[x_{i}^{+}, y_{i}^{+}\right] \stackrel{\text { dn }}{\longrightarrow}\left[x_{i}^{-}, \quad y_{i}^{-}\right]
$$

and the same with $j$ instead of $i$, if necessary. For example, if both $\mathfrak{r}_{i-1} \stackrel{\text { up }}{\rightarrow} \mathfrak{r}_{i}$ and $\mathfrak{r}_{j} \stackrel{\text { up }}{\rightarrow} \mathfrak{r}_{j+1}$ are up congruence-perspectivities, then we replace (5.17) by

$$
\left[x_{i}^{-}, y_{i}^{-}\right] \stackrel{\text { up }}{\rightarrow} D^{\prime}\left[x_{i}^{+}, y_{i}^{+}\right] \rightarrow D^{\prime} \ldots \rightarrow D^{\prime}\left[x_{j}^{+}, y_{j}^{+}\right] .
$$

This way, the number of new intervals in (5.6) decreases at least by one, which is a contradiction. Thus, we have shown that Subcase 3 a cannot occur, that is, no new interval occurs in (5.6). 


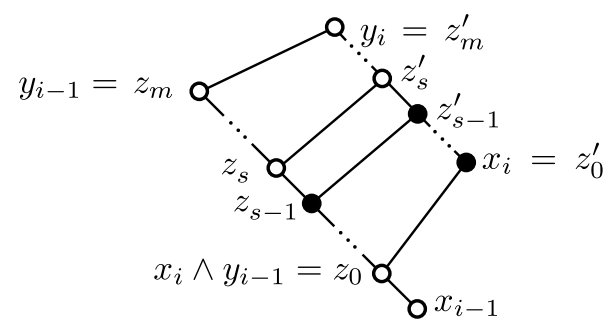

Figure 7. Illustration for Subcase 3b

Subcase 3b. $\mathfrak{r}_{i}$ is a [new,old] interval. If $\mathfrak{r}_{i-1} \stackrel{\text { dn }}{\rightarrow} D^{\prime} \mathfrak{r}_{i}$, then $x_{i}=x_{i-1} \wedge y_{i}$ implies that $x_{i} \in D$, which contradicts the initial assumption of Subcase 3b. Hence, $\mathfrak{r}_{i-1} \stackrel{\text { up }}{\longrightarrow} D^{\prime} \mathfrak{r}_{i}$. We take a maximal chain

$$
x_{i} \wedge y_{i-1}=z_{0} \prec z_{1} \prec \cdots \prec z_{m}=y_{i-1}
$$

in the interval $\left[x_{i} \wedge y_{i-1}, y_{i-1}\right]$, see Figure 7. Define $z_{j}^{\prime}:=z_{j} \vee x_{i}$ for $j=$ $0, \ldots, m$. By semimodularity, $x_{i}=z_{0}^{\prime} \preceq z_{1}^{\prime} \preceq \cdots \preceq z_{m}^{\prime}=y_{i}$. Since $z_{0}^{\prime}=x_{i}$ is a new element but $z_{m}^{\prime}=y_{i}$ is an old one, there is a subscript $s \in\{1, \ldots, m\}$ such that $z_{s-1}^{\prime}$ is new, $z_{s}^{\prime}$ is old, and $z_{s-1}^{\prime} \prec z_{s}^{\prime}$. Since $z_{s}^{\prime}=z_{s} \vee z_{s-1} \vee x_{i}=z_{s} \vee z_{s-1}^{\prime}$, the covering relations imply that $\left[z_{s-1}, z_{s}\right]$ and $\left[z_{s-1}^{\prime}, z_{s}^{\prime}\right]$ are transposed (and, therefore, congruence-equivalent) prime intervals and $\left[z_{s-1}, z_{s}\right] \stackrel{\text { up }}{\rightarrow} D^{\prime}\left[z_{s-1}^{\prime}, z_{s}^{\prime}\right]$. It follows from G. Czédli and E. T. Schmidt [11, Lemma 2.9] that $\left[z_{s-1}, z_{s}\right]$ and $\left[z_{s-1}^{\prime}, z_{s}^{\prime}\right]$ belong to the same trajectory of $D^{\prime}$. This implies easily, see Figure 2, that $z_{s-1}$ is new. Therefore, $\left[x_{i-1}, z_{s-1}\right]$ is an [old,new] subinterval of $\mathfrak{r}_{i-1}$. Thus, we have reached (5.13), competing the proof of Theorem 5.5.

Now, we are in the position to prove Theorem 4.4.

Proof of Theorem 4.4. By Theorem 3.7, it suffices to show that the statement holds for distributive slim rectangular diagrams, and its validity is inherited by multi-fork extensions at distributive 4-cells.

First, assume that $D$ is a distributive slim diagram. (Rectangularity is not needed in this paragraph.) We know from G. Czédli and E. T. Schmidt [12, Lemma 15] that $D$ contains no cover-preserving $\mathrm{S}_{7}$ sublattice. The absence of $\mathbf{S}_{7}$ sublattices implies that $D$ has no hat-trajectory. Thus $\tau$, given in Definition 4.3, is the equality relation. Therefore, if $n$ denotes the length of $D$, then $\left\langle\operatorname{Traj}(D) ; \leq_{\tau}\right\rangle$ is the $n$-element antichain. It is well-known that $\langle\mathrm{Ji}(\operatorname{Con} D) ; \leq\rangle$ is also an $n$-element antichain; this is trivial for chains, and the rest of slim distributive diagrams are reduced to chains by G. Czédli [3, Lemma 5.4] and G. Czédli and E. T. Schmidt [12, Theorem 11]. (Note that the main result of G. Grätzer and E. Knapp [22] also implies that $\langle\mathrm{Ji}(\operatorname{Con} D) ; \leq\rangle$ is an antichain, but here we also need the equality $|\operatorname{Ji}(\operatorname{Con} D)|=n$.) Since any two antichains of the same size are isomorphic, we can pick an order isomorphism $\psi:\langle\operatorname{Ji}(\operatorname{Con} D) ; \leq\rangle \rightarrow\left\langle\operatorname{Traj}(D) ; \leq_{\tau}\right\rangle$. Consider the surjective map $\varphi: \operatorname{PrInt}(D) \rightarrow \mathrm{Ji}(\operatorname{Con} D)$, defined by $\mathfrak{p} \mapsto \operatorname{con}(\mathfrak{p})$. Obviously, $\varphi$ is a coloring, 
and its kernel extends the equivalence $\sim^{\text {traj }}$. Since

$$
|\varphi(\operatorname{PrInt}(D))|=|\operatorname{Ji}(\operatorname{Con} D)|=n=|\operatorname{Traj}(D)|=\left|\operatorname{PrInt}(D) / \sim^{\text {traj }}\right|,
$$

we conclude that the kernel of $\varphi$ equals $\sim^{\text {traj }}$. This implies that $\xi$ from Definition 4.3(iv) equals $\psi \circ \varphi$, and we conclude that $\xi$ is a quasi-coloring, in fact, a coloring.

Next, assume that $H$ is a distributive 4-cell of a slim rectangular diagram $D_{0}, n \in \mathbf{N}, D=D_{0}\left[\mathrm{~S}_{7}^{(n)} \leadsto H\right]$, and $\tau_{0}=$ quor $\left(\sigma_{0}\right)$ is the quasiordering on $\operatorname{Traj}\left(D_{0}\right)$ according to Definition 4.3 , applied to $D_{0}$, such that the trajectory quasi-coloring $\xi_{0}: \operatorname{PrInt}\left(D_{0}\right) \rightarrow\left\langle\operatorname{Traj}\left(D_{0}\right) ; \tau_{0}\right\rangle$, given in Definition 4.3 , is a quasi-coloring. We have to show that $\xi: \operatorname{PrInt}(D) \rightarrow\langle\operatorname{Traj}(D) ; \tau\rangle$, given in Definition 4.3 for $D$, is a quasi-coloring. To simplify the notation, let $D_{1}=\mathrm{S}_{7}^{(n)}$, let $\tau_{1}$ be the quasiordering on $\operatorname{Traj}\left(D_{1}\right)$ defined by Figure 5 , and let $\xi_{1}: \operatorname{PrInt}\left(D_{1}\right) \rightarrow\left\langle\operatorname{Traj}\left(D_{1}\right) ; \tau_{1}\right\rangle$ be the trajectory quasi-coloring given in Lemma 5.1; note that $\xi_{1}$ is a quasi-coloring.

For $i \in\{0,1\}$, we define a map $\varphi_{i}: \operatorname{Traj}\left(D_{i}\right) \rightarrow \operatorname{Traj}(D)$ by the rule $\varphi_{i}(u)=$ $v$ iff the trajectories $u \in \operatorname{Traj}\left(D_{i}\right)$ and $v \in \operatorname{Traj}(D)$ have a prime interval $\mathfrak{p}$ in common. (We shall soon prove that $\varphi_{i}$ is a map.) Let $a_{0}$ and $b_{0}$ denote the trajectories of $D_{0}$ containing $\left[\operatorname{lc}(H), 1_{H}\right]$ and $\left[\operatorname{rc}(H), 1_{H}\right]$, respectively. Also, let $a_{1}$ and $b_{1}$ denote the trajectories of $D_{1}$ containing the same prime intervals, which are $\left[\operatorname{lc}\left(D_{1}\right), 1_{D_{1}}\right]$ and $\left[\operatorname{rc}\left(D_{1}\right), 1_{D_{1}}\right]$, respectively. Interrupting the proof of Theorem 4.4, we formulate an auxiliary statement.

Claim 5.8. Both $\varphi_{0}$ and $\varphi_{1}$ are injective maps, $\varphi_{0}\left(a_{0}\right)=\varphi_{1}\left(a_{1}\right)$, and $\varphi_{0}\left(b_{0}\right)=$ $\varphi_{1}\left(b_{1}\right)$. Also, we have that $\varphi_{0}\left(\operatorname{Traj}\left(D_{0}\right)\right) \cap \varphi_{1}\left(\operatorname{Traj}\left(D_{1}\right)\right)=\left\{\varphi_{i}\left(a_{i}\right), \varphi_{i}\left(b_{i}\right)\right\}$ for $i \in\{0,1\}$. Furthermore, $\varphi_{0}\left(\operatorname{Traj}\left(D_{0}\right)\right) \cup \varphi_{1}\left(\operatorname{Traj}\left(D_{1}\right)\right)=\operatorname{Traj}(D)$, that is, $\varphi_{0} \cup \varphi_{1}$ is surjective.

Proof. First, we prove that, for $i \in\{0,1\}, \varphi_{i}$ is a map. Assume that $u \in$ $\operatorname{Traj}\left(D_{i}\right), \mathfrak{p}_{1}, \mathfrak{p}_{2} \in u$, and $v_{1}, v_{2} \in \operatorname{Traj}(D)$ such that $\mathfrak{p}_{j} \in v_{j}$ for $j \in\{1,2\}$. We have that $\mathfrak{p}_{1} \sim^{\operatorname{traj}} D_{i} \mathfrak{p}_{2}$ in $D_{i}$, and we want to conclude that $\mathfrak{p}_{1} \sim^{\operatorname{traj}}{ }_{D} \mathfrak{p}_{2}$. (This is trivial for $i=1$, and it would be trivial for $i=0$ if $D_{0}$ was a coverpreserving sublattice of $D$, but this is not the case.) We know from G. Czédli and E. T. Schmidt [11, Lemma 2.9] that there exists a prime interval $\mathfrak{q}$ in $D_{i}$ such that both $\mathfrak{p}_{1}$ and $\mathfrak{p}_{2}$ are transposed up to $\mathfrak{q}$ in $D_{i}$. Since they are also transposed up to $\mathfrak{q}$ in $D$ and they belong to $\operatorname{PrInt}(D)$, because of $\mathfrak{p}_{j} \in v_{j}$, the semimodularity of $D$ gives that $\mathfrak{q}$ belongs to $\operatorname{PrInt}(D)$. Hence, applying [11, Lemma 2.9] in the opposite direction, we obtain that $\mathfrak{p}_{1} \sim^{\operatorname{traj}}{ }_{D} \mathfrak{p}_{2}$, which implies that $v_{1}=v_{2}$. This proves that $\varphi_{i}$ is a map from $\operatorname{Traj}\left(D_{i}\right)$ to $\operatorname{Traj}(D)$, for $i \in\{0,1\}$.

Next, we prove the injectivity of $\varphi_{i}$. For the sake of contradiction, suppose that $u_{1}, u_{2} \in \operatorname{Traj}\left(D_{i}\right), u_{1} \neq u_{2}$, and $\varphi_{i}\left(u_{1}\right)=v=\varphi_{i}\left(u_{2}\right)$. By definition, there exist $\mathfrak{p}_{1}, \mathfrak{p}_{2} \in \operatorname{PrInt}(D) \cap \operatorname{PrInt}\left(D_{i}\right)$ such that $\mathfrak{p}_{j} \in u_{j} \cap v$ for $j \in\{1,2\}$. Since $\mathfrak{p}_{1}, \mathfrak{p}_{2}$ belong to the same trajectory $v$ of $D$, [11, Lemma 2.9] gives a prime interval $\mathfrak{q} \in \operatorname{PrInt}(D)$ such that both $\mathfrak{p}_{1}$ and $\mathfrak{p}_{2}$ are transposed up to $\mathfrak{q}$ in $D$. 
If $\mathfrak{q} \in \operatorname{PrInt}\left(D_{i}\right)$, then $\mathfrak{p}_{1}$ and $\mathfrak{p}_{2}$ are transposed up to the same prime interval of $D_{i}$, so $\mathfrak{p}_{1} \sim^{\text {traj }} D_{i} \mathfrak{p}_{2}$ by [11, Lemma 2.9], which contradicts the equality $u_{1}=u_{2}$. Hence, $\mathfrak{q} \in \operatorname{PrInt}(D) \backslash \operatorname{PrInt}\left(D_{i}\right)$.

First, consider the case $i=0$. It is clear by the construction of $D=$ $D_{0}\left[\mathrm{~S}_{7}^{(n)} \leadsto H\right]$, see Figure 2, that if $\left.\mathfrak{q} \in \operatorname{PrInt}(D) \backslash \operatorname{PrInt}\left(D_{0}\right)\right)$ transposes down to an old prime interval, then $\mathfrak{q}$ is a parallel chain in the sense given right before (5.14), $\left[0_{\mathfrak{q}}^{-}, 1_{\mathfrak{q}}^{-}\right]$(see $(5.15)$ for its definition) equals $\left[0_{\mathfrak{q}} \wedge 0_{H}, 1_{\mathfrak{q}} \wedge 0_{H}\right]$, and $\left[0_{\mathfrak{q}}^{-}, 1_{\mathfrak{q}}^{-}\right]$also transposes down to the old prime interval in question. In particular, $\left[0_{\mathfrak{q}}^{-}, 1_{\mathfrak{q}}^{-}\right]$transposes down to $\mathfrak{p}_{1}$ and $\mathfrak{p}_{2}$, whence $\mathfrak{p}_{1} \sim{ }^{\operatorname{traj}} D_{0} \mathfrak{p}_{2}$ by $[11$, Lemma 2.9]. This contradicts $u_{1} \neq u_{2}$ and proves that $\varphi_{0}$ is injective.

Second, consider the case $i=1$. Since $\mathfrak{p}_{1}, \mathfrak{p}_{2} \in \operatorname{PrInt}(D) \cap \operatorname{PrInt}\left(D_{1}\right)=$ $\left\{\left[\operatorname{lc}(H), 1_{H}\right],\left[\operatorname{rc}(H), 1_{H}\right]\right\}$ and $u_{1} \neq u_{2}$ gives $\mathfrak{p}_{1} \neq \mathfrak{p}_{2}$, we can assume that $\mathfrak{p}_{1}=\left[\operatorname{lc}(H), 1_{H}\right]$ and $\mathfrak{p}_{2}=\left[\operatorname{rc}(H), 1_{H}\right]$. Since $v \in \operatorname{Traj}(D)$ contains both $\mathfrak{p}_{1}$ and $\mathfrak{p}_{2}$, [11, Lemma 2.9] yields an $\mathfrak{r} \in \operatorname{PrInt}(D)$ such that $\mathfrak{p}_{1}$ and $\mathfrak{p}_{2}$ are transposed up to $\mathfrak{r}$ in $D$. Since $0_{\mathfrak{r}} \geq 0_{\mathfrak{p}_{1}} \vee 0_{\mathfrak{p}_{2}}=\operatorname{lc}(H) \vee \operatorname{rc}(H)=1_{H}$, we obtain that $\mathfrak{r} \in \operatorname{PrInt}\left(D_{0}\right)$. This, together with [11, Lemma 2.9], implies that $\left[\operatorname{lc}(H), 1_{H}\right]$ and $\left[\operatorname{rc}(H), 1_{H}\right]$ belongs to the same trajectory $v_{0}$ of $D_{0}$. We know from (5.2) that $1_{H}$ has exactly two lower covers, $\mathrm{lc}(H)$ and $\mathrm{rc}(H)$, in $D_{0}$. Therefore, the trajectory $v_{0}$, when leaving $H$ to the right, goes upwards. Similarly, when it arrives at $H$ from the left, it goes downwards. This contradicts (2.1), which proves the injectivity of $\varphi_{1}$.

The surjectivity of $\varphi_{0} \cup \varphi_{1}$ is obvious by the construction of $D$. Clearly,

$$
\varphi_{0}\left(\operatorname{Traj}\left(D_{0}\right)\right) \cap \varphi_{1}\left(\operatorname{Traj}\left(D_{1}\right)\right) \supseteq\left\{\varphi_{0}\left(a_{0}\right), \varphi_{0}\left(b_{0}\right)\right\}=\left\{\varphi_{1}\left(a_{1}\right), \varphi_{1}\left(b_{1}\right)\right\} .
$$

Since each member of $\operatorname{Traj}(D)$ departs from the left boundary chain of $D$, we have that $\mid \operatorname{Traj}(D)]=\operatorname{length}(D)$. Similarly, $\left.\mid \operatorname{Traj}\left(D_{i}\right)\right]=\operatorname{length}\left(D_{i}\right)$ for $i \in$ $\{0,1\}$. Clearly, length $(D)=$ length $\left(D_{0}\right)+\operatorname{length}\left(D_{1}\right)-2$. Thus $|\operatorname{Traj}(D)|=$ $\left|\operatorname{Traj}\left(D_{0}\right)\right|+\left|\operatorname{Traj}\left(D_{1}\right)\right|-2$. This, together with the injectivity of $\varphi_{0}$ and $\varphi_{1}$ and the surjectivity of $\varphi_{0} \cup \varphi_{1}$, implies that $\left|\varphi_{0}\left(\operatorname{Traj}\left(D_{0}\right)\right) \cap \varphi_{1}\left(\operatorname{Traj}\left(D_{1}\right)\right)\right|=2$. Consequently, the inclusion in (5.19) is an equality, proving our claim.

Now, we return to the proof of Theorem 4.4. We are going to use Theorem 5.5 as follows. Let $\varphi_{i}\left(\tau_{i}\right)=\left\{\left\langle\varphi_{i}(x), \varphi_{i}(y)\right\rangle: x \leq_{\tau_{i}} y\right\}$ for $i \in\{0,1\}$. We have assumed that $\xi_{0}: \operatorname{Pr} \operatorname{Int}\left(D_{0}\right) \rightarrow\left\langle\operatorname{Traj}\left(D_{0}\right) ; \tau_{0}\right\rangle$ is a quasi-coloring. Hence, so is $\varphi_{0} \circ \xi_{0}: \operatorname{PrInt}\left(D_{0}\right) \rightarrow\left\langle\varphi_{0}\left(\operatorname{Traj}\left(D_{0}\right)\right) ; \varphi_{0}\left(\tau_{0}\right)\right\rangle$, because $\varphi_{0}$ is injective by Claim 5.8. We let $A=\varphi_{0}\left(\operatorname{Traj}\left(D_{0}\right)\right), B=\varphi_{1}\left(\operatorname{Traj}\left(D_{1}\right)\right)$, and $C=\operatorname{Traj}(D)$. We know from Claim 5.8 that $C=A \cup B$. Instead of the quasi-coloring $\xi_{1}: \operatorname{PrInt}\left(\mathrm{S}_{7}^{(n)}\right)=\operatorname{PrInt}\left(D_{1}\right) \rightarrow\left\langle\operatorname{Traj}\left(D_{1}\right) ; \tau_{1}\right\rangle$, the injectivity of $\varphi_{1}$ allows us to consider the quasi-coloring

$$
\varphi_{1} \circ \xi_{1}: \operatorname{PrInt}\left(D_{1}\right) \rightarrow\left\langle\varphi_{1}\left(\operatorname{Traj}\left(D_{1}\right)\right) ; \varphi_{1}\left(\tau_{1}\right\rangle\right) .
$$

With the new setting $\left\langle\varphi_{0} \circ \xi_{0}, \varphi_{1} \circ \xi_{1}\right\rangle$ instead of $\langle\gamma, \xi\rangle$, the satisfaction of (5.5) follows from Claim 5.8. Therefore, all the stipulations of Definition 5.4 hold 
for the new setting. Hence, letting

$$
\eta=\operatorname{quor}\left(\varphi_{0}\left(\tau_{0}\right) \cup \varphi_{1}\left(\tau_{1}\right)\right),
$$

Theorem 5.5 implies that $\delta: \operatorname{PrInt}(D) \rightarrow\langle\operatorname{Traj}(D) ; \eta\rangle$ is a quasi-coloring. Here $\delta$ is determined by Definition 5.4, applied to the present situation. However, it is easy to see that $\delta$ is the same as $\xi$. Therefore, our task is only to prove that $\eta=\tau$. As a preparation for this task, we claim that, for $u \neq v \in \operatorname{Traj}(D)$,

$$
\text { if } u \leq_{\varphi_{1}\left(\tau_{1}\right)} v \text {, then } u \leq_{\sigma} v \text { and, consequently, } u \leq_{\tau} v \text {. }
$$

To prove this, choose $u_{1}, v_{1} \in \operatorname{Traj}\left(D_{1}\right)$ such that $u=\varphi_{1}\left(u_{1}\right), v=\varphi_{1}\left(v_{1}\right)$, and $u_{1} \leq_{\tau_{1}} v_{1}$. Clearly, $u_{1} \neq v_{1}$. Thus, since the structure of $D_{1}=\mathrm{S}_{7}^{(n)}$ is quite simple by Lemma 5.1 and Figure 1 , we easily conclude that $u_{1} \leq_{\sigma_{1}} v_{1}$. Also, the understanding of the structure of $D_{1}$ implies that $u_{1}=c_{m}$ for some $m \in\{1, \ldots, n\}$. Hence, $\varphi_{1}$ preserves the top edge $\left[s_{m}, 1_{D_{1}}\right]$ of $u_{1}$, that is, $\mathfrak{h}(u)=\mathfrak{h}\left(\varphi_{1}\left(u_{1}\right)\right)=\mathfrak{h}\left(u_{1}\right)$. If it also preserves the top edge of $v_{1}$, then we clearly obtain $u \leq_{\sigma} v$, as desired. Hence, we assume that $\mathfrak{h}\left(v_{1}\right) \neq \mathfrak{h}(v)$. Up to left-right symmetry, this is only possible if $\mathfrak{h}\left(v_{1}\right)=\left[\mathrm{rc}\left(D_{1}\right), 1_{D_{1}}\right]=\left[\mathrm{rc}(H), 1_{H}\right]$. Let $v_{0} \in \operatorname{Traj}\left(D_{0}\right)$ denote the trajectory of $D_{0}$ through $\left[\operatorname{rc}(H), 1_{H}\right]$; note that $\varphi_{0}\left(v_{0}\right)=v$. It follows from $(5.2)$ that $v_{0}$ goes upwards at $\left[\operatorname{rc}(H), 1_{H}\right]$. Thus, by $(2.1)$, it reaches its top edge on the right of $\left[\mathrm{rc}(H), 1_{H}\right]$. Since $D$ and $D_{0}$ are different only in $\downarrow 1_{H}$ and (2.1) also applies to $v$ in $D$, we conclude that $\mathfrak{h}(v)=\mathfrak{h}\left(v_{0}\right)$ and that the section of $v \in \operatorname{Traj}(D)$ from $\left[\operatorname{rc}(H), 1_{H}\right]$ to $\mathfrak{h}(v)$ and that of $v_{0} \in \operatorname{Traj}\left(D_{0}\right)$ from $\left[\mathrm{rc}(H), 1_{H}\right]$ to $\mathfrak{h}\left(v_{0}\right)$ are the same. In the interval $\left[\mathrm{rc}(H), 1_{\mathfrak{h}(v)}\right]$, this common section is an up-trajectory. Hence, we easily conclude that $1_{H} \wedge 0_{\mathfrak{h}(v)}=\operatorname{rc}(H)$ and $1_{H} \vee 0_{\mathfrak{h}(v)}=1_{\mathfrak{h}(v)}$. In particular, $1_{\mathfrak{h}(u)}=1_{H} \leq 1_{\mathfrak{h}(v)}$ and $1_{H} \not \leq 0_{\mathfrak{h}(v)}$. Consequently, we obtain that $0_{\mathfrak{h}(u)}=$ $0_{\mathfrak{h}\left(u_{1}\right)}=s_{m} \not \leq 0_{\mathfrak{h}(v)}$. Consequently, $u \leq_{\sigma} v$, which proves (5.21).

Next, we assert that, for $u \neq v \in \operatorname{Traj}(D)$,

$$
\text { if } u \leq_{\varphi_{0}\left(\tau_{0}\right)} v \text {, then } u \leq_{\tau} v \text {. }
$$

Assume that $u \leq_{\varphi_{0}\left(\tau_{0}\right)} v$. Then there are $u_{0}, v_{0} \in \operatorname{Traj}\left(D_{0}\right)$ such that $u=$ $\varphi_{0}\left(u_{0}\right), v=\varphi_{0}\left(v_{0}\right)$, and $u_{0} \leq_{\tau_{0}} v_{0}$. This means that there is an $e \in \mathbb{N}$ and there are pairwise distinct trajectories $w_{0}=u_{0}, w_{1}, \ldots, w_{e}=v_{0}$ of $D_{0}$ such that $w_{i-1} \leq_{\sigma_{0}} w_{i}$ for $i \in\{1, \ldots, e\}$. It is clear from the construction of $D=D_{0}\left[\mathrm{~S}_{7}^{(n)} \leadsto H\right]$ that $\varphi_{0}$ and, under a reasonable restriction, $\varphi_{1}$ preserve the top edges. It is also clear that $\varphi_{0}$ preserves straightness and non-straightness. We summarize this for further reference:

$$
\begin{aligned}
& \text { if } w_{0} \in \operatorname{Traj}\left(D_{0}\right) \text { and } w_{1} \in \operatorname{Traj}\left(D_{1}\right) \backslash\left\{a_{1}, b_{1}\right\} \text {, then } \\
& \mathfrak{h}\left(\varphi_{0}\left(w_{0}\right)\right)=\mathfrak{h}\left(w_{0}\right), \mathfrak{h}\left(\varphi_{1}\left(w_{1}\right)\right)=\mathfrak{h}\left(w_{1}\right), \text { and } w_{0} \text { is a } \\
& \text { straight trajectory iff so is } \varphi_{0}\left(w_{0}\right) .
\end{aligned}
$$

In particular, $\mathfrak{h}\left(w_{i}\right)=\mathfrak{h}\left(\varphi_{0}\left(w_{i}\right)\right)$ for $i \in\{1, \ldots, e\}$. Hence, since $D_{0}$ is a sublattice of $D$, it follows by 4.3 (ii) that $\varphi_{0}\left(w_{i-1}\right) \leq_{\sigma} \varphi_{0}\left(w_{i}\right)$. Hence, $u=$ $\varphi_{0}\left(u_{0}\right)=\varphi_{0}\left(w_{0}\right) \leq_{\sigma} \ldots \leq_{\sigma} \varphi_{0}\left(w_{e}\right)=\varphi_{0}\left(v_{0}\right)=v$, which gives $u \leq_{\tau} v$, as claimed. This proves (5.22). 
Now, from (5.20), (5.21), and (5.22), we conclude that $\eta \subseteq \tau$.

By definition, $\tau=$ quor $(\sigma)$. Therefore, in order to prove the converse inclusion $\tau \subseteq \eta$, it suffices to show that $\sigma \subseteq \eta$. Assume that $u \neq v \in \operatorname{Traj}(D) \operatorname{such}$ that $u \leq_{\sigma} v$. We have to show that $u \leq_{\eta} v$. The assumption $u \leq_{\sigma} v$ implies that $u$ is a hat-trajectory of $D$. By Claim 5.8, the trajectories $u$ and $v$ are " $\varphi_{0} \cup \varphi_{1}$-images", and there are four cases to consider.

First, assume that $u=\varphi_{0}\left(u_{0}\right)$ and $v=\varphi_{0}\left(v_{0}\right)$ for some $u_{0}, v_{0} \in \operatorname{Traj}\left(D_{0}\right)$. It follows from (5.23) that $u_{0} \leq_{\sigma_{0}} v_{0}$. Hence $u \leq_{\varphi_{0}\left(\sigma_{0}\right)} v$, which yields that $u \leq_{\eta} v$, as desired.

Second, assume that $u=\varphi_{1}\left(u_{1}\right)$ and $v=\varphi_{1}\left(v_{1}\right)$ for some trajectories $u_{1}, v_{1} \in \operatorname{Traj}\left(D_{1}\right) \backslash\left\{a_{1}, b_{1}\right\}$. Note that $u_{1}, v_{1} \in\left\{c_{1}, \ldots, c_{n}\right\}$. Clearly, $u_{1} \leq_{\sigma_{1}} v_{1}$. This gives that $u \leq_{\varphi_{1}\left(\sigma_{1}\right)} v$, implying that $u \leq_{\eta} v$.

Third, assume that $u=\varphi_{0}\left(u_{0}\right)$ and $v=\varphi_{1}\left(v_{1}\right)$ for some $u_{0}=\operatorname{Traj}\left(D_{0}\right)$ and $v_{1} \in \operatorname{Traj}\left(D_{1}\right) \backslash\left\{a_{1}, b_{1}\right\}$. Note that $v_{1} \in\left\{c_{1}, \ldots, c_{n}\right\}$. Observe that $u_{0}$ is a hat-trajectory by (5.23). We also know from (5.23) that $\mathfrak{h}\left(u_{0}\right)=\mathfrak{h}(u)$ and $\mathfrak{h}\left(v_{1}\right)=\mathfrak{h}(v)$. This, together with $u \leq_{\sigma} v$, yields that $1_{\mathfrak{h}\left(u_{0}\right)}=1_{\mathfrak{h}(u)} \leq$ $1_{\mathfrak{h}(v)}=1_{\mathfrak{h}\left(v_{1}\right)}=1_{H}$. Thus $\downarrow 1_{H}$, taken in $D_{0}$, contains the top edge $\mathfrak{h}\left(u_{0}\right)$ of the hat-trajectory $u_{0} \in \operatorname{Traj}\left(D_{0}\right)$. Therefore, $1_{\mathfrak{h}\left(u_{0}\right)}$ violates $(5.2)$, and we obtain that this case cannot occur.

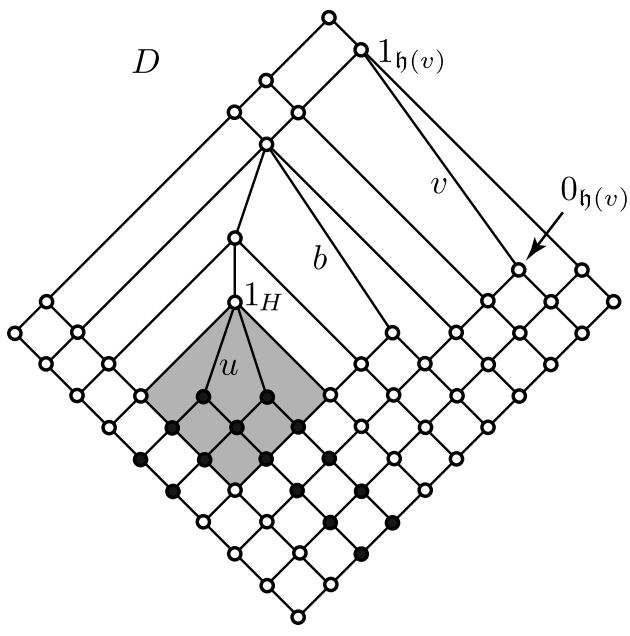

Figure 8. Case of $u=\varphi_{1}\left(u_{1}\right)$ and $v=\varphi_{0}\left(v_{0}\right)$

Fourth, assume that $u=\varphi_{1}\left(u_{1}\right)$ and $v=\varphi_{0}\left(v_{0}\right)$ for some trajectories $u_{1}$ in $\operatorname{Traj}\left(D_{1}\right) \backslash\left\{a_{1}, b_{1}\right\}$ and $v_{0}$ in $\operatorname{Traj}\left(D_{0}\right)$. The situation is depicted in Figure 8, where $D_{0}=D \backslash$ black-filled elements $\}, H$ is the light-grey 4-cell of $D_{0}$, and the trajectories are labeled at their top edges. We will use (5.23) implicitly. Since $u \leq_{\sigma} v$, we have that $1_{H}=1_{\mathfrak{h}\left(u_{1}\right)}=1_{\mathfrak{h}(u)} \leq 1_{\mathfrak{h}(v)}$. Therefore, again by $u \leq_{\sigma} v$, we obtain that $0_{\mathfrak{h}(u)} \not \leq 0_{\mathfrak{h}(v)}$. If we had $1_{H} \leq 0_{\mathfrak{h}(v)}$, then we would obtain a contradiction by $0_{\mathfrak{h}(u)} \leq 1_{\mathfrak{h}(u)}=1_{H} \leq 0_{\mathfrak{h}(v)}$. Hence, $1_{H} \not \leq 0_{\mathfrak{h}(v)}$, 
which implies that $1_{H}>1_{H} \wedge 0_{\mathfrak{h}(v)}=1_{H} \wedge 0_{\mathfrak{h}\left(v_{0}\right)} \in D_{0}$. Consequently, it follows from (5.2) that $1_{H} \wedge 0_{\mathfrak{h}(v)} \leq \operatorname{lc}(H)$ or $1_{H} \wedge 0_{\mathfrak{h}(v)} \leq \operatorname{rc}(H)$. By left-right symmetry, we can assume that

$$
1_{H} \wedge 0_{\mathfrak{h}(v)} \leq \operatorname{rc}(H) .
$$

The trajectory of $D$ containing $\left[\operatorname{rc}(H), 1_{H}\right]$ is denoted by $b=\varphi_{1}\left(b_{1}\right)=$ $\varphi_{0}\left(b_{0}\right)$. Since $u_{1} \leq_{\sigma_{1}} b_{1}$, we have that $u \leq_{\varphi_{1}\left(\sigma_{1}\right)} b$. This, together with $\sigma_{1} \subseteq \tau_{1}$ gives that that $u \leq_{\varphi_{1}\left(\tau_{1}\right)} b$, which yields that $u \leq_{\eta} b$. Since $0_{\mathfrak{h}(v)} \prec 1_{\mathfrak{h}(v)}$ and $1_{H} \not \leq 0_{\mathfrak{h}(v)}$ but $1_{H} \leq 1_{\mathfrak{h}(v)}$, we have that $1_{H} \vee 0_{\mathfrak{h}(v)}=1_{\mathfrak{h}(v)}$. If the inequality in $(5.24)$ is an equality, then $\left[\operatorname{rc}(H), 1_{H}\right]$ and $\left[0_{\mathfrak{h}(v)}, 1_{\mathfrak{h}(v)}\right]$ are transposed intervals, $v=b$, and we obtain $u \leq_{\eta} b=v$, as desired.

Hence, we can assume that (5.24) is a strict inequality. However, even in this case it is clear that $\left\langle\operatorname{rc}(H), 1_{H}\right\rangle \in \operatorname{con}_{D_{0}}\left(0_{\mathfrak{h}(v)}, 1_{\mathfrak{h}(v)}\right)=\operatorname{con}_{D_{0}}\left(0_{\mathfrak{h}\left(v_{0}\right)}, 1_{\mathfrak{h}\left(v_{0}\right)}\right)$, that is, $\operatorname{con}_{D_{0}}\left(\operatorname{rc}(H), 1_{H}\right)$ is a subset of $\operatorname{con}_{D_{0}}\left(0_{\mathfrak{h}\left(v_{0}\right)}, 1_{\mathfrak{h}\left(v_{0}\right)}\right)$. Hence, using the assumption that $\xi_{0}$ is a quasi-coloring, we obtain that $b_{0}=\xi_{0}\left(\operatorname{rc}(H), 1_{H}\right) \leq_{\tau_{0}}$ $\xi_{0}\left(0_{\mathfrak{h}\left(v_{0}\right)}, 1_{\mathfrak{h}\left(v_{0}\right)}\right)=v_{0}$. This gives that $b \leq_{\varphi_{0}\left(\tau_{0}\right)} v$, and we conclude that $b \leq_{\eta} v$. Combining this with $u \leq_{\eta} b$, we obtain that $u \leq_{\eta} v$, as claimed. This completes the proof of Theorem 4.4 .

\section{From multi-fork extensions to patch extensions}

Recall that multi-fork extensions are special cases of patch extensions. Now, we are in the position to generalize the two main lemmas from the previous section. For a patch extension $D[P \leadsto H]$, define the retraction $\psi: D[P \leadsto H] \rightarrow$ $D$ by the congruence $\boldsymbol{\alpha}$ whose non-singleton blocks are depicted by dotted closed curves in Figure 2. (Although the figure gives only a single example, the general definition of $\boldsymbol{\alpha}$ should be straightforward.) So, $\psi(x)$ is defined as the largest element in the $\boldsymbol{\alpha}$-block of $x$.

Lemma 6.1 (Patch version of the retraction lemma). If His a distributive 4-cell of a slim semimodular lattice diagram $D$ and $P$ is a patch diagram, then the retraction map $\psi: D[P \sim H] \rightarrow D$ defined above is a lattice homomorphism.

Proof. We prove the lemma by induction on the size of $P$. If $|P|=4$, then the statement is trivial since $D[P \leadsto H]=D$ and $\psi$ is the identity map. Next, assume that $|P|>4$, and that the lemma is true for all patch diagrams of smaller size. By Theorem 3.7, $P$ is of the form $P=Q\left[\mathrm{~S}_{7}^{(n)} \leadsto G\right]$, where $Q$ is a patch diagram and $G$ is a distributive 4-cell of $Q$. Clearly, $G$ is also a distributive 4 -cell of $D[P \leadsto H]$. It is straightforward to verify that $D[P \leadsto H]=(D[Q \leadsto H])\left[S_{7}^{(n)} \leadsto G\right]$; the tedious details are omitted. By the induction hypothesis, the retraction map $\psi_{0}: D[Q \sim H] \rightarrow D$ is a lattice homomorphism. We know from Lemma 5.3 that so is the retraction map $\psi_{1}:(D[Q \leadsto H])\left[\mathrm{S}_{7}^{(n)} \sim G\right] \rightarrow D[Q \sim H]$. Hence, the composite map $\psi_{0} \circ \psi_{1}$, from $D[P \sim H]=(D[Q \sim H])\left[\mathrm{S}_{7}^{(n)} \sim G\right]$ to $D$, is also a lattice homomorphism. Finally, it is straightforward to see that $\psi=\psi_{0} \circ \psi_{1}$. 
Next, we generalize the multi-fork theorem. Let $H$ be a distributive 4-cell of a slim semimodular lattice diagram $D$, and let $P$ be a patch lattice diagram. We denote by $D^{\prime}$ the patch extension $D[P \sim H]$. Let $\gamma: \operatorname{PrInt}(D) \rightarrow\langle A ; \nu\rangle$ be a quasi-coloring, and let $\xi: \operatorname{Pr} \operatorname{Int}(P) \rightarrow\left\langle\operatorname{Traj}(P) ; \leq_{\tau}\right\rangle$ be the trajectory quasicoloring of $P$. (We know from Theorem 4.4 that $\xi$ is a quasi-coloring.) Let $B=$ $\operatorname{Traj}(P)$, and assume that $\gamma\left(\operatorname{lc}(H), 1_{H}\right)=a=\xi\left(\operatorname{lc}(P), 1_{P}\right), \gamma\left(\operatorname{rc}(H), 1_{H}\right)=$ $b=\xi\left(\operatorname{rc}(P), 1_{P}\right)$, and $A \cap B=\{a, b\}$. On the set $C=A \cup B$, we define $\eta=$ quor $(\nu \cup \tau)$. Also, we define a map $\delta: \operatorname{PrInt}\left(D^{\prime}\right) \rightarrow\langle C ; \eta\rangle$ by the following two obvious rules. First, $\delta$ should extend $\gamma \cup \xi$. Second, if $\delta(\mathfrak{p})$ is not determined by the first rule, then take a $\mathfrak{q} \in \operatorname{PrInt}\left(D^{\prime}\right)$, nearest to $\mathfrak{p}$ with $1_{\mathfrak{q}} \geq 1_{\mathfrak{p}}$, such that $\mathfrak{p} \sim^{\text {traj }} D^{\prime} \mathfrak{q}$ in $D^{\prime}$ and $\delta(\mathfrak{q})$ is defined, and let $\delta(\mathfrak{p})=\delta(\mathfrak{q})$. (Note at this point that if dropped the stipulation that $\mathfrak{q}$ is nearest to $\mathfrak{p}$ in the trajectory of $\mathfrak{p}$ in $D^{\prime}$, then $\delta$ would not be uniquely defined but the following lemma would still hold for every choice of $\delta$.) For technical reasons, we denote $\delta$ by $\gamma \triangleleft \xi$.

Lemma 6.2 (Patch lemma). With the assumptions in the paragraph above, $\delta$ is a quasi-coloring.

Proof. We adopt the notation, the assumptions, and the already established facts of the proof of Lemma 6.1. In particular, $|P|>4, P=Q\left[\mathrm{~S}_{7}^{(n)} \sim G\right]$, and $D^{\prime}=D[P \sim H]=(D[Q \sim H])\left[\mathrm{S}_{7}^{(n)} \leadsto G\right]$. Let $\xi_{0}, \xi_{1}$, and $\xi$ be the trajectory quasi-colorings of $\mathrm{S}_{7}^{(n)}, Q$, and $P$, respectively. It is straightforward to check that $\delta=\gamma \triangleleft \xi$ equals $\left(\gamma \triangleleft \xi_{1}\right) \triangleleft \xi_{0}$. Let $\delta_{1}=\gamma \triangleleft \xi_{1}$. It is a quasi-coloring by the induction hypothesis. Hence, so is $\delta=\delta_{1} \triangleleft \xi_{0}$ by Theorem 5.5.

\section{Trajectory colorings and the main result}

Combining Theorem 4.4 and Lemma 4.1 for a slim rectangular lattice $L$, we can obviously obtain a representation of $\langle\mathrm{Ji}(\operatorname{Con} L) ; \leq\rangle$. If we take G. Czédli $[3$, Lemma 2.1] into account, we can clearly obtain a coloring for $L$ from its trajectory quasi-coloring. Actually, we give the same coloring below; however, we do it in a more explicite and useful way. We begin with a couple of "twin definitions"; the coincidence of their notation is on purpose and will not cause confusion.

Definition 7.1. Let $D$ be a slim rectangular diagram.

(i) For $u, v \in \operatorname{Traj}(D)$, we let $\langle u, v\rangle \in \Theta$ iff $u=v$, or both $u$ and $v$ are hat trajectories such that $1_{\mathfrak{h}(u)}=1_{\mathfrak{h}(v)}$. The quotient set $\operatorname{Traj}(D) / \Theta$ of $\operatorname{Traj}(D)$ by the equivalence $\Theta$ is denoted $\widehat{\operatorname{Traj}}(D)$. Its elements are denoted by $u / \Theta$, where $u \in \operatorname{Traj}(D)$.

(ii) On the set $\widehat{\operatorname{Traj}}(D)$, we define a relation $\widehat{\sigma}$ as follows. For $u / \Theta$ and $v / \Theta$ in $\widehat{\operatorname{Traj}}(D)$, we let $\langle u / \Theta, v / \Theta\rangle \in \widehat{\sigma}$ iff $u / \Theta \neq v / \Theta$ and there exist $u^{\prime}, v^{\prime} \in \operatorname{Traj}(D)$ such that $\left\langle u, u^{\prime}\right\rangle,\left\langle v, v^{\prime}\right\rangle \in \Theta$ and $u^{\prime} \leq_{\sigma} v^{\prime}$. (Recall that $\sigma$ is given in Definition 4.3.)

(iii) We let $\widehat{\tau}=\operatorname{quor}(\widehat{\sigma})$, the reflexive transitive closure of $\widehat{\sigma}$ on $\widehat{\operatorname{Traj}}(D)$. 
(iv) The trajectory coloring of $D$ is the coloring $\widehat{\xi}$ from $\operatorname{Pr} \operatorname{Int}(D)$ onto the ordered set $\langle\widehat{\operatorname{Traj}}(D) ; \widehat{\tau}\rangle$, defined by the rule that $\widehat{\xi}(\mathfrak{p})$ is the $\Theta$-block of the unique trajectory containing $\mathfrak{p}$.

We will soon prove that $\widehat{\xi}$ is a coloring. This definition determines a lattice concept, that is, it does not matter which planar diagram of a given slim rectangular lattice is considered. Its "twin brother" below is formulated for lattices. Thus, we should note that if $L$ is a slim rectangular lattice, then G. Czédli and E. T. Schmidt [14, Lemma 4.7] implies that its planar diagram is unique apart from reflection by a vertical axis. Hence, the interior of $L$ is uniquely defined. For $x \in$ Mi $L$, the unique cover of $x$ is denoted by $x^{*}$.

Definition 7.2. Let $L$ be slim a rectangular lattice.

(i) We define an equivalence relation on $\mathrm{Mi} L$ as follows. For $x, y \in \mathrm{Mi} L$, let $\langle x, y\rangle \in \Theta$ mean that $x=y$, or both $x$ and $y$ are in the interior of $L$ and $x^{*}=y^{*}$. The quotient set $\operatorname{Mi} L / \Theta$ is denoted $\widehat{\operatorname{Mi}} L$. For $x \in \operatorname{Mi} L$, we denote the $\Theta$-block of $x$ by $x / \Theta$.

(ii) We define a relation $\widehat{\sigma}$ on $\widehat{\mathrm{Mi}} L$ by the rule $\langle x / \Theta, y / \Theta\rangle \in \widehat{\sigma}$ iff $x / \Theta \neq y / \Theta$, $x$ is in the interior of $L, x^{*} \leq y^{*}$, but there are $x^{\prime} \in x / \Theta$ and $y^{\prime} \in y / \Theta$ such that $x^{\prime} \not y^{\prime}$.

(iii) We let $\widehat{\tau}=\operatorname{quor}(\widehat{\sigma})$, the reflexive transitive closure of $\widehat{\sigma}$ on $\widehat{\mathrm{Mi}} L$.

Now, we are in the position to formulate the main result of the paper. It gives a structural description for the congruence lattice of a slim rectangular lattice.

Theorem 7.3. Let $L$ be a slim rectangular lattice, and let $D$ be a planar diagram of $L$.

(i) $\langle\widehat{\operatorname{Traj}}(D) ; \widehat{\tau}\rangle$ from Definition 7.1 is an ordered set, and it is isomorphic to $\langle\mathrm{Ji}(\mathrm{Con} L) ; \leq\rangle$. Furthermore, $\widehat{\xi}$ in Definition $7.1(\mathrm{iv})$ is a coloring.

(ii) $\langle\widehat{\mathrm{Mi}} L ; \widehat{\tau}\rangle$ from Definition 7.2 is an ordered set, and it is isomorphic to $\langle\mathrm{Ji}(\operatorname{Con} L) ; \leq\rangle$.

We illustrate Theorem 7.3 with Figure 4 , where $\operatorname{Mi} D=\{a, b, \ldots, k, \ell\}$ consists of the black-filled elements, and $L$ is the lattice determined by $D$.

Proof of Theorem 7.3. First, we prove (i). By Lemma 4.1, Theorem 4.4, and G. Czédli [3, Lemma 2.1], it suffices to show that $\langle\widehat{\operatorname{Traj}}(D), \widehat{\tau}\rangle$ is the ordered set associated with $\langle\operatorname{Traj}(D) ; \tau\rangle$. Using Theorem 3.7 and Theorem 5.5, we prove this by induction.

First, assume that $D$ is a slim distributive diagram of length $n$. The second paragraph in the proof of Theorem 4.4 explicitely says that both $\langle\mathrm{Ji}(\operatorname{Con} D)$; $\leq$ \rangle and $\langle\operatorname{Traj}(D) ; \tau\rangle$ are $n$-element antichains. Since distributivity does not permit hat-trajectories by, say, (5.2), we obtain that $\Theta$ is the equality relation, $\sigma=\varnothing$, and $\widehat{\tau}$ is the equality relation. Therefore, $\langle\widehat{\operatorname{Traj}}(;) \widehat{\tau}\rangle$ is also an $n$-element antichain, and the statement for $D$ follows trivially. 
Next, assume that the statement holds for a slim rectangular diagram $D_{0}$, $H$ is a distributive 4-cell of $D_{0}$, and $D=D_{0}\left[\mathrm{~S}_{7}^{(n)} \leadsto H\right]$. Let $D_{1}$ stand for $\mathrm{S}_{7}^{(n)}$. Let $\Psi=\tau \cap \tau^{-1}$ be the equivalence induced by $\tau$, see also (4.1). The relations associated with $D_{0}$ and $D_{1}$ are subscripted with 0 and 1 . We adopt the notation of Claim 5.8, and we shall use (the multi-fork) Theorem 5.5 for the situation described in and right above (5.20). Note, however, that $\eta$ in (5.20) is actually $\tau$; this is what the second part of the proof of Theorem 4.4 after Claim 5.8 yields. The new trajectories $\varphi_{1}\left(c_{1}\right), \ldots, \varphi_{1}\left(c_{n}\right)$ that arrive with $\mathrm{S}_{7}^{(n)}$ are the trajectories through $\left[s_{1}, 1_{H}\right], \ldots,\left[s_{n}, 1_{H}\right]$; see Figure 2 . It follows easily from Definition 5.4, Lemma 5.1, and Claim 5.8 that two trajectories of $D$ are rarely $\varphi_{1}\left(\sigma_{1}\right)$-related; in fact, the only possibilities are the following: $\varphi_{1}\left(c_{i}\right) \leq_{\varphi_{1}\left(\sigma_{1}\right)} \varphi_{1}\left(c_{j}\right)$ with $i \neq j, \varphi_{1}\left(c_{i}\right) \leq_{\varphi_{1}\left(\sigma_{1}\right)} \varphi_{1}\left(a_{1}\right)=\varphi_{0}\left(a_{0}\right)$, and $\varphi_{1}\left(c_{i}\right) \leq \varphi_{1}\left(\sigma_{1}\right) \varphi_{1}\left(b_{1}\right)=\varphi_{0}\left(b_{0}\right)$. Hence, taking $\tau=\operatorname{quor}\left(\varphi_{0}\left(\tau_{0}\right) \cup \varphi_{1}\left(\tau_{1}\right)\right)=$ quor $\left(\varphi_{0}\left(\sigma_{0}\right) \cup \varphi_{1}\left(\sigma_{1}\right)\right)$ and (5.5) (tailored to the present situation) into account, it follows in a straightforward way that, for arbitrary $u_{0}, v_{0} \in \operatorname{Traj}\left(D_{0}\right)$,

$$
\varphi_{0}\left(u_{0}\right) \leq_{\tau} \varphi_{0}\left(v_{0}\right) \Longleftrightarrow \varphi_{0}\left(u_{0}\right) \leq_{\varphi_{0}\left(\tau_{0}\right)} \varphi_{0}\left(v_{0}\right) .
$$

This implies that, for $u_{0}, v_{0} \in \operatorname{Traj}\left(D_{0}\right)$,

$$
\left\langle\varphi_{0}\left(u_{0}\right), \varphi_{0}\left(v_{0}\right)\right\rangle \in \Psi \quad \Longleftrightarrow\left\langle u_{0}, v_{0}\right\rangle \in \Psi_{0}=\tau_{0} \cap \tau_{0}^{-1} .
$$

Next, to show that $\Psi=\Theta$, assume that $u, v \in \operatorname{Traj}(D)$ such that $\langle u, v\rangle$ is in $\Psi$. We obtain from (the multi-fork) Theorem 5.5 that either $u, v$ belongs to $\left\{\varphi_{1}\left(c_{1}\right), \ldots, \varphi_{1}\left(c_{n}\right)\right\}$, or $u=\varphi_{0}\left(u_{0}\right)$ and $v=\varphi_{0}\left(v_{0}\right)$ for some $u_{0}, v_{0} \in$ $\operatorname{Traj}\left(D_{0}\right)$. In the first case, $\langle u, v\rangle \in \Theta$ is obvious. In the second case, $\left\langle u_{0}, v_{0}\right\rangle \in$ $\Psi_{0}$ by (7.1). Thus the induction hypothesis gives that $\left\langle u_{0}, v_{0}\right\rangle \in \Theta_{0}$. Hence, we conclude that $\langle u, v\rangle \in \Theta$ by (5.23). Therefore, $\Psi \subseteq \Theta$.

To prove the converse inclusion, assume that $\langle u, v\rangle \in \Theta$ but $u \neq v$. If $u=\varphi_{0}\left(u_{0}\right)$ and $v=\varphi_{0}\left(v_{0}\right)$ for some $u_{0}, v_{0} \in \operatorname{Traj}\left(D_{0}\right)$, then $\left\langle u_{0}, v_{0}\right\rangle \in \Theta_{0}$ by (5.23). Thus the induction hypothesis gives that $\left\langle u_{0}, v_{0}\right\rangle \in \Psi_{0}$, and we obtain the desired $\langle u, v\rangle \in \Psi$ from (7.1). Hence, we can assume that, say, $u$ is not of the form $\varphi_{0}\left(u_{0}\right)$ with $u_{0} \in \operatorname{Traj}\left(D_{0}\right)$. Thus $u \in\left\{\varphi_{1}\left(c_{1}\right), \ldots, \varphi_{1}\left(c_{n}\right)\right\}$. Since $H$ is a distributive 4-cell of $D_{0}$, there is no $v_{0} \in \operatorname{Traj}\left(D_{0}\right)$ with $1_{\mathfrak{h}\left(v_{0}\right)}=1_{H}=$ $1_{\mathfrak{h}(u)}$. Hence (5.23) yields that there is no $v_{0} \in \operatorname{Traj}\left(D_{0}\right)$ with $\left\langle\varphi_{0}\left(v_{0}\right), u\right\rangle \in \Theta$. Therefore, $v$ also belongs to $\left\{\varphi_{1}\left(c_{1}\right), \ldots, \varphi_{n}\left(c_{n}\right)\right\}$, whence $\langle u, v\rangle$ and $\langle v, u\rangle$ belong to $\varphi_{1}\left(\tau_{1}\right) \subseteq \tau$, and thus $\langle u, v\rangle \in \Psi$, as claimed. This completes the argument proving that $\Psi=\Theta$.

Therefore, Traj $(D)$ is the underlying set of the ordered set associated with $\langle\operatorname{Traj}(D) ; \tau\rangle$. From now on, we write $\Theta$ for $\Psi$. Let $\tau^{\bullet}$ denote the relation that (4.1) associates with $\tau$. That is, for $u, v \in \operatorname{Traj}(L),\langle u / \Theta, v / \Theta\rangle \in \tau^{\bullet}$ iff $u \leq_{\tau} v$. To complete the proof of (i), we have to show that $\tau^{\bullet}=\widehat{\tau}$. Let $u / \Theta, v / \Theta \in \widehat{\operatorname{Traj}}(D)$, that is, let $u, v \in \operatorname{Traj}(D)$. We can assume that $u / \Theta \neq v / \Theta$.

Assume first that $\langle u / \Theta, v / \Theta\rangle \in \tau^{\bullet}$. Then $u \leq_{\tau} v$, and we have a sequence $u=w_{0} \leq_{\sigma} w_{1} \leq_{\sigma} \ldots \leq_{\sigma} w_{k}=v$ in $\operatorname{Traj}(D)$. Since $\left\langle w_{i-1} / \Theta, w_{i-1} / \Theta\right\rangle \in \widehat{\sigma}$ or 
$w_{i-1} / \Theta=w_{i-1} / \Theta$ for $i \in\{1, \ldots, k\}$, we obtain that $\langle u / \Theta, v / \Theta\rangle \in \widehat{\tau}$. That is, $\tau^{\bullet} \subseteq \widehat{\tau}$.

Next, to prove the converse inclusion, assume that $\langle u / \Theta, v / \Theta\rangle \in \widehat{\tau}$. Then there exists a sequence $w_{0}, \ldots, w_{k} \in \operatorname{Traj}(D)$ such that $u / \Theta=w_{0} / \Theta, v / \Theta=$ $w_{k} / \Theta$, and $\left\langle w_{i-1} / \Theta, w_{i} / \Theta\right\rangle \in \widehat{\sigma}$ for $i \in\{1, \ldots, k\}$. By 7.1(ii), there are appropriate $w_{i}^{-}$and $w_{i}^{+}$in $\operatorname{Traj}(D)$ such that

$$
u \Theta w_{0} \Theta w_{0}^{+} \leq_{\sigma} w_{1}^{-} \Theta w_{1} \Theta w_{1}^{+} \leq_{\sigma} w_{2}^{-} \Theta w_{2} \Theta w_{2}^{+} \leq_{\sigma} \cdots \Theta w_{k} \Theta v .
$$

Since both $\sigma$ and $\Theta=\Psi$ are included in $\tau$, which is transitive, (7.2) yields that $u \leq_{\tau} v$. Hence, $\langle u / \Theta, v / \Theta\rangle \in \tau^{\bullet}$. This proves the equality $\widehat{\tau}=\tau^{\bullet}$ and statement (i) of the theorem.

In order to prove statement (ii), it suffices to show that it is just a reformulation of statement (i). To do so, observe that if $x \in \operatorname{Mi} L$, then the trajectory containing $\left[x, x^{*}\right]$ arrives upwards at $\left[x, x^{*}\right]$ from the left, and leaves $\left[x, x^{*}\right]$ downwards to the right. This easy fact, together with (2.1), implies that $\left[x, x^{*}\right]$ is the top edge of its trajectory. Conversely, the presence of a coverpreserving $S_{7}$ and planarity imply that if $[x, y]$ is the top edge of a trajectory, then $x \in \operatorname{Mi} L$. Thus the map $\zeta: \operatorname{Traj}(L) \rightarrow \operatorname{Mi} L$, defined by $\zeta(u)=0_{\mathfrak{h}(u)}$, is a bijection. Furthermore,

$$
u \text { is a hat-trajectory iff } \zeta(u) \text { is in the interior of } L \text {. }
$$

Hence, it follows by comparing the twin definitions, 7.1 and 7.2 , that $\zeta$ translates (i) into (ii).

\section{Remarks and generalizations}

Remark 8.1. Unfortunately, the counterpart of Lemma 5.3 and that of Theorem 5.5, that is, [3, Lemma 4.5] and [3, Lemma 5.1], are incorrect statements in G. Czédli [3], since the distributivity of the 4-cells in question was not assumed. However, this does not affect the main result of [3], because [3, Lemma $5.1]$ is only used at distributive 4-cells, where we can replace it by Theorem 5.5 , and [3, Lemma 4.5] is only used to prove [3, Lemma 5.1].

Next, to point out that the scope of Theorem 4.4 is much larger than the class of slim rectangular lattices, we need the following definition. The middle element $s_{1}$ of $\mathrm{S}_{7}$ is defined by Figure 1 .

Definition 8.2. Let $\mathcal{K}$ denote the class of finite slim semimodular lattices $L$ with the following property: for every $x, s \in L$, if $s$ is the middle element $s_{1}$ of a cover-preserving $\mathrm{S}_{7}$ sublattice, $x<s$, and $[x, s]$ is a chain, then $x \notin \mathrm{Mi} L$.

A straightforward induction based on Theorem 3.7 yields that every slim rectangular lattice belongs to $\mathcal{K}$. The smallest slim semimodular lattice not in $\mathcal{K}$ is obtained from $S_{7}^{(2)}$, see Figure 1 , by deleting $s_{0}=w_{\ell}$ and $w_{\ell} \wedge s_{1}$. The single-fork variant of the following statement can be extracted from G. Czédli 
and E. T. Schmidt [12], because a lattice in $\mathcal{K}$ cannot contain weak forks (defined there). Therefore, the proof of Theorem 3.7 applies, and we obtain the following result.

Proposition 8.3. Each lattice in $\mathcal{K}$ can be obtained from a slim distributive lattice by a sequence of multi-fork extensions at distributive 4-cells. Moreover, every lattice obtained this way belongs to $\mathcal{K}$.

The proof of Theorem 4.4 only uses rectangularity once, where it recalls Theorem 3.7; now we can recall Claim 8.3. Thus, we obtain the following proposition. Remember that Theorem 4.4 is a "lattice statement", that is, the choice of the diagram of a given lattice is irrelevant. Therefore, Definition 4.3 is also meaningful for lattices instead of diagrams.

Proposition 8.4. If $L \in \mathcal{K}$, then $\xi$ from Definition 4.3 is a quasi-coloring of $L$.

Remark 8.5. There is another way to extend the scope of Theorem 4.4, which is motivated by G. Grätzer and E. Knapp [23, Theorem 7] and its proof. We know from G. Czédli and E. T. Schmidt [12, Lemma 21] that each slim semimodular lattice can be obtained from a slim rectangular lattice by deleting (strong) corners. The deletion of a corner does not really change the quasi-coloring by the corner lemma in G. Czédli [3, Lemma 5.4], and does not change the trajectories too much. Hence, in principle, arbitrary slim semimodular lattices can be traced back to the scope of Theorem 4.4.

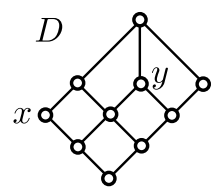

Figure 9. Two meet-irreducible elements in a rectangular diagram

Finally, Figure 9 explains why we have to distinguish boundary and interior elements in Definition 7.2 (or straight trajectories and hat-trajectories in Definition 4.3): we can have that $x^{*}<y^{*}$ and $x \not \leq y$, but $\operatorname{con}\left(x, x^{*}\right) \nsubseteq \subseteq \operatorname{con}\left(y, y^{*}\right)$.

\section{REFERENCES}

[1] Czédli, G.: The matrix of a slim semimodular lattice. Order 29, 85-103 (2012)

[2] Czédli, G.: Coordinatization of join-distributive lattices. Algebra Universalis 71, 385-404 (2014) arXiv: 1208.3517

[3] Czédli, G.: Representing homomorphisms of distributive lattices as restrictions of congruences of rectangular lattices. Algebra Universalis 67, 313-345 (2012)

[4] Czédli, G.: The asymptotic number of planar, slim, semimodular lattice diagrams. Order (submitted); arXiv: 1206.3679

[5] Czédli, G.: Finite convex geometries of circles. Discrete Mathematics (submitted); arXiv: 1212.3456 
[6] Czédli, G.: Quasiplanar diagrams and slim semimodular lattices. Order (submitted); arXiv: 1212.6904

[7] Czédli, G., Dékány, T., Ozsvárt, L., Szakács, N., Udvari, B.: On the number of slim, semimodular lattices. Mathematica Slovaca (submitted); arXiv: 1208.6173

[8] Czédli, G., Grätzer, G.: Notes on planar semimodular lattices. VII. Resections of planar semimodular lattices. Order (in press); DOI 10.1007/s11083-012-9281-1

[9] Czédli, G., Grätzer, G.: Planar semimodular lattices and their diagrams. In: Grätzer, G., Wehrung, F. (eds.) Lattice Theory: Special Topics and Applications. Birkhäuser Verlag, Basel (2013, in press)

[10] Czédli, G., Ozsvárt, L., Udvari, B.: How many ways can two composition series intersect?. Discrete Mathematics 312, 3523-3536 (2012)

[11] Czédli, G., Schmidt, E.T.: The Jordan-Hölder theorem with uniqueness for groups and semimodular lattices. Algebra Universalis 66, 69-79 (2011)

[12] Czédli, G., Schmidt, E.T.: Slim semimodular lattices. I. A visual approach. Order 29, 481-497 (2012)

[13] Czédli, G., Schmidt, E.T.: Composition series in groups and the structure of slim semimodular lattices. Acta Sci. Math. (Szeged) (in press); arXiv:1208.4749

[14] Czédli, G., Schmidt, E.T.: Slim semimodular lattices. II. A description by patchwork systems. Order, DOI: 10.1007/s11083-012-9271-3 (Published online August 29, 2012)

[15] Grätzer, G.: General Lattice Theory, 2nd edn. Birkhäuser, Basel (1998)

[16] Grätzer, G.: The Congruences of a Finite Lattice. A Proof-by-picture Approach. Birkhäuser, Boston (2006)

[17] Grätzer, G.: Lattice Theory: Foundation. Birkhäuser, Basel (2011)

[18] Grätzer, G.: Notes on planar semimodular lattices. VI. On the structure theorem of planar semimodular lattices. Algebra Universalis 69, 301-304 (2013)

[19] Grätzer, G.: A technical lemma for congruences of finite lattices. Algebra Universalis (submitted)

[20] Grätzer, G.: Congruences of fork extensions of lattices. Acta Sci. Math. (Szeged) (submitted); arXiv:1307.8404

[21] Grätzer, G., Knapp, E.: Notes on planar semimodular lattices. I. Construction. Acta Sci. Math. (Szeged), 73, 445-462 (2007)

[22] Grätzer, G., Knapp, E.: Notes on planar semimodular lattices. II. Congruences. Acta Sci. Math. (Szeged), 74, 23-36 (2008)

[23] Grätzer, G., Knapp, E.: Notes on planar semimodular lattices. III. Congruences of rectangular lattices. Acta Sci. Math. (Szeged), 75, 29-48 (2009)

[24] Grätzer, G., Knapp, E.: Notes on planar semimodular lattices. IV. The size of a minimal congruence lattice representation with rectangular lattices. Acta Sci. Math. (Szeged), 76, 3-26 (2010)

[25] Grätzer, G., Lakser, H., Schmidt, E.T.: Congruence lattices of finite semimodular lattices. Canad. Math. Bull. 41, 290-297 (1998)

[26] Grätzer, G., Schmidt, E.T.: A short proof of the congruence representation theorem for semimodular lattices. arXiv: 1303.4464

[27] Jakubík, J.: Congruence relations and weak projectivity in lattices. Časopis Pěst. Mat. 80, 206-216 (1955) (Slovak)

[28] Kelly, D., Rival, I.: Planar lattices. Can. J. Math. 27, 636-665 (1975)

[29] Schmidt, E.T.: Congruence lattices and cover preserving embeddings of finite length semimodular lattices. Acta Sci. Math. Szeged 77, 47-52 (2011)

\section{GÁBOR CZÉdLI}

University of Szeged, Bolyai Institute. Szeged, Aradi vértanúk tere 1, HUNGARY 6720 e-mail: czedli@math.u-szeged.hu URL: http://www.math.u-szeged.hu/ czedli/ 